\title{
Constraining composite Higgs models using LHC data
}

\author{
Avik Banerjee, ${ }^{a}$ Gautam Bhattacharyya, ${ }^{a}$ Nilanjana Kumar $^{a}$ and Tirtha Sankar Ray $^{b}$ \\ ${ }^{a}$ Saha Institute of Nuclear Physics, HBNI, \\ 1/AF Bidhan Nagar, Kolkata 700064, India \\ ${ }^{b}$ Department of Physics and Centre for Theoretical Studies, \\ Indian Institute of Technology Kharagpur, \\ Kharagpur 721302, India \\ E-mail: avik.banerjeesinp@saha.ac.in, \\ gautam.bhattacharyya@saha.ac.in, nilanjana.kumar@saha.ac.in, \\ tirthasankar.ray@gmail.com
}

ABstract: We systematically study the modifications in the couplings of the Higgs boson, when identified as a pseudo Nambu-Goldstone boson of a strong sector, in the light of LHC Run 1 and Run 2 data. For the minimal coset $\mathrm{SO}(5) / \mathrm{SO}(4)$ of the strong sector, we focus on scenarios where the standard model left- and right-handed fermions (specifically, the top and bottom quarks) are either in $\mathbf{5}$ or in the symmetric $\mathbf{1 4}$ representation of $\mathrm{SO}(5)$. Going beyond the minimal $\mathbf{5}_{\mathbf{L}}-\mathbf{5}_{\mathbf{R}}$ representation, to what we call here the 'extended' models, we observe that it is possible to construct more than one invariant in the Yukawa sector. In such models, the Yukawa couplings of the $125 \mathrm{GeV}$ Higgs boson undergo nontrivial modifications. The pattern of such modifications can be encoded in a generic phenomenological Lagrangian which applies to a wide class of such models. We show that the presence of more than one Yukawa invariant allows the gauge and Yukawa coupling modifiers to be decorrelated in the 'extended' models, and this decorrelation leads to a relaxation of the bound on the compositeness scale ( $f \geq 640 \mathrm{GeV}$ at $95 \% \mathrm{CL}$, as compared to $f \geq 1 \mathrm{TeV}$ for the minimal $\mathbf{5}_{\mathbf{L}}-\mathbf{5}_{\mathbf{R}}$ representation model). We also study the Yukawa coupling modifications in the context of the next-to-minimal strong sector coset $\mathrm{SO}(6) / \mathrm{SO}(5)$ for fermion-embedding up to representations of dimension 20. While quantifying our observations, we have performed a detailed $\chi^{2}$ fit using the ATLAS and CMS combined Run 1 and available Run 2 data.

Keywords: Beyond Standard Model, Higgs Physics, Technicolor and Composite Models ARXIV EPRINT: 1712.07494 


\section{Contents}

1 Introduction 1

2 Composite models and modified Yukawa couplings 3

$2.1 \mathrm{SO}(5) / \mathrm{SO}(4)$ coset 3

$2.2 \mathrm{SO}(6) / \mathrm{SO}(5)$ coset 6

$\begin{array}{lll}3 & \text { Effective phenomenological Lagrangian } & 7\end{array}$

4 Constraints from LHC Run 1 and Run 2 Higgs data 10

5 Conclusions $\quad 12$

A Fermion embeddings $\quad 14$

$\begin{array}{lll}\text { A.1 } & \mathrm{SO}(5) / \mathrm{SO}(4) \text { coset } & 14\end{array}$

$\begin{array}{lll}\text { A.2 } & \mathrm{SO}(6) / \mathrm{SO}(5) \text { coset } & 15\end{array}$

$\begin{array}{ll}\text { B Details of form factors } & 15\end{array}$

$\begin{array}{ll}\text { C } \text { I-functions for next-to-minimal models } & 17\end{array}$

$\begin{array}{ll}\text { D Expressions for } \Delta_{t}, \delta_{t} \text { and } \Delta_{t}^{\eta} & 17\end{array}$

\section{Introduction}

With increasing precision in measurements of the Higgs boson properties at the Large Hadron Collider (LHC), the possibility that the Higgs may be a composite object [1-5] can be put to stringent tests. In this context, the scenarios where the Higgs is identified as a pseudo Nambu-Goldstone boson (pNGB) of a strongly interacting sector are of special interest. This has received considerable attention following its identification as a holographic dual of 5d gauge-Higgs unification models [6-9]. In this paper, however, we stick to an effective $4 \mathrm{~d}$ scenario, and do not comment on possible UV completion of such models. The approximate shift-symmetry of the pNGBs can screen the weak scale from physics beyond the compositeness scale $(f \sim \mathcal{O}(\mathrm{TeV}))$. This provides a well-motivated framework for natural electroweak symmetry breaking.

The direct signatures of these models at the LHC could be the appearance of additional resonances of the strong sector [10-18]. However taking cue from non-observation of these resonances, attempts have been made to push up the resonance masses while keeping the theory still natural [19-23]. The other inevitable and testable features of these models are deviations of the Higgs couplings compared to their standard model (SM) predictions. 
One of the consequences of compositeness is that the couplings are replaced by form factors which are momentum dependent. However, it is difficult to test this momentum dependence at the LHC. Nevertheless, the nonlinearity of the pNGB dynamics provides a finite shift in the Higgs couplings measurable in the precision era of the LHC. In this paper we make a systematic study of the pattern and constraints on such modifications that arise in a general class of composite Higgs models.

We categorize the scenarios considered under three major heads:

- Minimal model: coset $\mathrm{SO}(5) / \mathrm{SO}(4)$, with both the left- and right-handed fermions kept in the fundamental 5 of $\mathrm{SO}(5)$, represented in literature as $\mathrm{MCHM}_{5_{L}-5_{R}}$ [24-29].

- Extended models: coset $\mathrm{SO}(5) / \mathrm{SO}(4)$, with at least one of the left- or right-handed fermions kept in the symmetric $\mathbf{1 4}$ of $\mathrm{SO}(5)$. They are denoted in literature as $\mathrm{MCHM}_{14_{L}-14_{R}}, \mathrm{MCHM}_{14_{L}-5_{R}}$, and $\mathrm{MCHM}_{5_{L}-14_{R}}$ [29-35].

- Next-to-minimal models: coset $\mathrm{SO}(6) / \mathrm{SO}(5)$, denoted as NMCHM, with different choices of representation up to dimension $\mathbf{2 0}$ [21, 36-44].

The couplings of the pNGB Higgs with the weak gauge bosons $(V V h)$ are usually suppressed in a general class of composite models. The parameter $\xi \equiv v^{2} / f^{2} \ll 1$, where $v=246 \mathrm{GeV}$ is the electroweak vacuum expectation value (vev), controls this suppression. The Yukawa couplings are generated through a mixing between the elementary fermions and the operators of the strong sector. Once the strong sector is integrated out the effective Higgs-fermion interaction term looks like [45, 46],

$$
\mathcal{L}_{\text {eff }} \propto \bar{f}_{L} H f_{R} \mathcal{F}\left(\frac{H^{\dagger} H}{f^{2}}\right)
$$

where $\mathcal{F}\left(H^{\dagger} H / f^{2}\right)$ is a function of the $\mathrm{SU}(2)_{\mathrm{L}}$ doublet Higgs field $(H)$. The contributions from the higher dimensional operators with independent coefficients, added to the SM dimension-4 Yukawa term, give rise to a modification in the couplings of the Higgs with the fermions $(f \bar{f} h)$, see also $[47,48]$ in a different context. In the minimal model, the SM fermions couple to only one operator of the strong sector. As a result the modification of the couplings depends on only one free parameter $\xi$. The other parameters in the effective Lagrangian are fixed from the requirement of reproducing the corresponding SM fermion mass. Therefore, $f \bar{f} h$ and $V V h$ couplings get highly correlated, and stringent constraints on $f$ emerge $[49,50]$ from the increasingly precise measurements of Higgs production and decays at LHC. In the extended models, owing to the presence of more than one invariant in the Yukawa sector with different coefficients, the correlation between $f \bar{f} h$ and $V V h$ modifiers is weakened, and we observe a possible relaxation of the bound on $f$. This happens in certain regions of the parameter space where a possible enhancement in $f \bar{f} h$ vertex can partially offset the suppression in $V V h$ coupling. Additionally, the extended 
models, carrying more than one invariant in the Yukawa sector, have the distinct advantage of being free from 'double tuning' [30]. ${ }^{1}$

In this paper, we first concentrate on a systematic and comparative study of various possibilities of Higgs coupling modifications in the context of the extended models. ${ }^{2}$ For each such possibilities, we construct one-loop Coleman-Weinberg Higgs potential [52], and identify regions of parameters space where the top mass, Higgs mass and the electroweak vev are reproduced. Next we consider the next-to-minimal model which contains a SM scalar-singlet $(\eta)$ apart from the Higgs doublet. Their mixing can significantly modify the observed Higgs boson couplings. In this context also, we survey different fermionic representations and calculate the corresponding modifications to Yukawa couplings.

We then construct an effective phenomenological Lagrangian whose parameters capture the coupling modifications of a general class of models mentioned earlier. The explicit connection between the coefficients of the Lagrangian and the parameters of the specific models is specified on a case-by-case basis. We perform a $\chi^{2}$ analysis with the ATLAS and CMS combined Run 1 [53] and available Run 2 data [54-66] to estimate a bound on $f$ in the extended models and compare it with that of the minimal model. In the context of the next-to-minimal model, we provide an estimate of the amount of doublet-singlet scalar mixing allowed by the current data.

The rest of the paper is organized as follows. In section 2 we review the consequences of various fermionic embedding for $\mathrm{SO}(5) / \mathrm{SO}(4)$ as well as $\mathrm{SO}(6) / \mathrm{SO}(5)$ cosets. In section 3 we present a phenomenological Lagrangian that captures the generic features of a wide class of models in terms of the Higgs coupling modifiers. Following this parametrization we perform a fit to the existing data using the $\chi^{2}$ minimization technique in section 4 . Finally, we draw our conclusions in section 5 .

\section{Composite models and modified Yukawa couplings}

In this section we consider different representations for fermions in $\mathrm{SO}(5) / \mathrm{SO}(4)$ and $\mathrm{SO}(6) / \mathrm{SO}(5)$ cosets and work out the modifications in the top quark Yukawa coupling in a systematic manner.

\section{$2.1 \mathrm{SO}(5) / \mathrm{SO}(4)$ coset}

As long as the coset is $\mathrm{SO}(5) / \mathrm{SO}(4)$, the modification in $V V h$ coupling is solely determined by $\xi$, as

$$
k_{V V h}=\frac{g_{V V h}}{\left(g_{V V h}\right)_{\mathrm{SM}}}=\sqrt{1-\xi} \simeq 1-\frac{1}{2} \xi .
$$

\footnotetext{
${ }^{1} \Delta=1 / \xi$ is a measure of minimal tuning in any composite Higgs model. On top of this, an additional tuning, dubbed 'double tuning', arises in scenarios (e.g. $\mathrm{MCHM}_{5_{L}-5_{R}}$ ) where the coefficients of the quadratic and quartic terms in the potential are not in the same order of the elementary-composite mixing parameter. This can be avoided when either the fermion kinetic and/or the Yukawa terms contain at least two invariants.

${ }^{2}$ We do not consider representation 10 of $\mathrm{SO}(5)$ because it does not lead to more than one Yukawa invariant keeping a discrete parity that protects the $Z b \bar{b}$ vertex $[29,51]$. Note that the choice $\mathrm{MCHM}_{14_{L}-1_{R}}$, where $t_{R}$ can be fully composite, involves minimal tuning as compared to the double tuned $\mathrm{MCHM}_{5_{L}-5_{R}}$ [30]. However, we do not consider this choice because it contains a single Yukawa invariant.
} 


\begin{tabular}{|c|c|c|c|}
\hline Models & $\Pi_{t_{L}}(q, h)$ & $\Pi_{t_{R}}(q, h)$ & $\Pi_{t_{L} t_{R}}(q, h)$ \\
\hline $\mathrm{MCHM}_{5_{\mathrm{L}}-5_{\mathrm{R}}}$ & $\Pi_{0}^{L}+\Pi_{1}^{L} \frac{h^{2}}{f^{2}}$ & $\Pi_{0}^{R}+\Pi_{1}^{R} \frac{h^{2}}{f^{2}}$ & $\Pi_{1}^{L R} \frac{h}{f} \sqrt{1-\frac{h^{2}}{f^{2}}}$ \\
\hline $\mathrm{MCHM}_{1_{\mathrm{L}}-14_{\mathrm{R}}}$ & $\Pi_{0}^{L}+\Pi_{1}^{L} \frac{h^{2}}{f^{2}}+\Pi_{2}^{L} \frac{h^{4}}{f^{4}}$ & $\Pi_{0}^{R}+\Pi_{1}^{R} \frac{h^{2}}{f^{2}}+\Pi_{2}^{R} \frac{h^{4}}{f^{4}}$ & $\frac{h}{f} \sqrt{1-\frac{h^{2}}{f^{2}}}\left(\Pi_{1}^{L R}+\Pi_{2}^{L R} \frac{h^{2}}{f^{2}}\right)$ \\
\hline $\mathrm{MCHM}_{14_{\mathrm{L}}-5_{\mathrm{R}}}$ & $\Pi_{0}^{L}+\Pi_{1}^{L} \frac{h^{2}}{f^{2}}+\Pi_{2}^{L} \frac{h^{4}}{f^{4}}$ & $\Pi_{0}^{R}+\Pi_{1}^{R} \frac{h^{2}}{f^{2}}$ & $\frac{h}{f}\left(\Pi_{1}^{L R}+\Pi_{2}^{L R} \frac{h^{2}}{f^{2}}\right)$ \\
\hline $\mathrm{MCHM}_{5_{\mathrm{L}}-14_{\mathrm{R}}}$ & $\Pi_{0}^{L}+\Pi_{1}^{L} \frac{h^{2}}{f^{2}}$ & $\Pi_{0}^{R}+\Pi_{1}^{R} \frac{h^{2}}{f^{2}}+\Pi_{2}^{R} \frac{h^{4}}{f^{4}}$ & $\frac{h}{f}\left(\Pi_{1}^{L R}+\Pi_{2}^{L R} \frac{h^{2}}{f^{2}}\right)$ \\
\hline
\end{tabular}

Table 1. List of $\Pi$-functions, defined in eq. (2.2) for different representations.

The number of Yukawa invariants, on the other hand, depends on the representations in which $t_{L}$ and $t_{R}$ are embedded. We write down the relevant invariants using the pNGB representation $\Sigma=\left(0,0,0, h / f, \sqrt{1-h^{2} / f^{2}}\right)^{T}$ in the unitary gauge:

- $t_{L}$ and $t_{R}$ in $\mathbf{5}\left(\mathrm{MCHM}_{5_{\mathrm{L}}-5_{\mathrm{R}}}\right): \quad\left(\bar{Q}_{t_{L}}^{5} \cdot \Sigma\right)\left(\Sigma^{T} \cdot T_{t_{R}}^{5}\right)$,

- $t_{L}$ and $t_{R}$ in $14\left(\mathrm{MCHM}_{14_{\mathrm{L}}-14_{\mathrm{R}}}\right): \Sigma^{T} \cdot \bar{Q}_{t_{L}}^{14} \cdot T_{t_{R}}^{14} \cdot \Sigma, \quad\left(\Sigma^{T} \cdot \bar{Q}_{t_{L}}^{14} \cdot \Sigma\right)\left(\Sigma^{T} \cdot T_{t_{R}}^{14} \cdot \Sigma\right)$,

- $t_{L}$ in 14, $t_{R}$ in $\mathbf{5}\left(\mathrm{MCHM}_{14_{\mathrm{L}}-5_{\mathrm{R}}}\right): \Sigma^{T} \cdot \bar{Q}_{t_{L}}^{14} \cdot T_{t_{R}}^{5},\left(\Sigma^{T} \cdot \bar{Q}_{t_{L}}^{14} \cdot \Sigma\right)\left(\Sigma^{T} \cdot T_{t_{R}}^{5}\right)$,

- $t_{L}$ in $\mathbf{5}, t_{R}$ in $14\left(\mathrm{MCHM}_{5_{\mathrm{L}}-14_{\mathrm{R}}}\right): \bar{Q}_{t_{L}}^{5} \cdot T_{t_{R}}^{14} \cdot \Sigma,\left(\bar{Q}_{t_{L}}^{5} \cdot \Sigma\right)\left(\Sigma^{T} \cdot T_{t_{R}}^{14} \cdot \Sigma\right)$.

Above, $Q_{t_{L}}$ and $T_{t_{R}}$ contain $t_{L}$ and $t_{R}$ as incomplete $\mathrm{SO}(5)$ multiplets, respectively (see appendix A.1). The most general Lagrangian involving the top quark can be written as

$$
\mathcal{L}=\bar{t}_{L} \phi \Pi_{t_{L}}(q, h) t_{L}+\bar{t}_{R} \phi \Pi_{t_{R}}(q, h) t_{R}+\bar{t}_{L} \Pi_{t_{L} t_{R}}(q, h) t_{R}+\text { h.c. }
$$

The dependence on the strong sector dynamics is encoded inside the momentum dependent $\Pi$-functions. In table 1 , we show the explicit forms of those functions for various representations in terms of the Higgs field with coefficients $\Pi_{0,1,2}^{L, R, L R}(q)$. The expressions for the latter in terms of the masses $\left(m_{i}\right)$ and decay constants $\left(F_{i}^{L, R}\right)$ of the strong sector

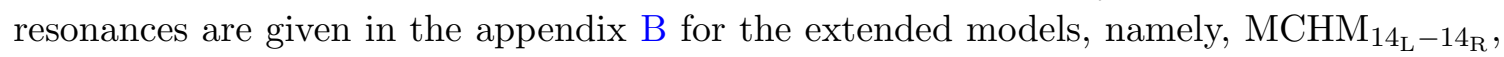
$\mathrm{MCHM}_{14_{\mathrm{L}}-5_{\mathrm{R}}}$ and $\mathrm{MCHM}_{5_{\mathrm{L}}-14_{\mathrm{R}}}$ respectively. The mass of the top quark and the modification of top Yukawa can be calculated from eq. (2.2) as

$$
m_{t}=\left.\frac{\left|\Pi_{t_{L} t_{R}}(q, h)\right|}{\sqrt{\Pi_{t_{L}}(q, h) \Pi_{t_{R}}(q, h)}}\right|_{q \rightarrow 0, h \rightarrow v}, \quad k_{t \bar{t} h}=\frac{y_{t \bar{t} h}}{\left(y_{t \bar{t} h}\right)_{\mathrm{SM}}}=\frac{1}{\left(y_{t \bar{t} h}\right)_{\mathrm{SM}}}\left(1-\frac{1}{2} \xi\right) \frac{\partial m_{t}}{\partial v} .
$$

In the second equality of eq. (2.3), the factor $\left(1-\frac{1}{2} \xi\right)$ arises due to canonical normalization of the Higgs field. As argued in [31, 67], the top quark contribution to the effective gluongluon-Higgs $(g g h)$ coupling in composite Higgs models is independent of the wave function renormalization effects of the top quark due to cancellation with resonance loops. This 
would imply a deviation in effective ggh coupling compared to the effective $t \bar{t} h$ coupling. The modification of the effective $g g h$ coupling can be expressed as

$$
k_{g g h}^{(t)}=\frac{c_{g g h}}{\left(c_{g g h}\right)_{\mathrm{SM}}}=\left.\frac{1}{\left(c_{g g h}\right)_{\mathrm{SM}}}\left(1-\frac{1}{2} \xi\right) \frac{\partial \log \left|\Pi_{t_{L} t_{R}}(q, h)\right|}{\partial h}\right|_{q \rightarrow 0, h \rightarrow v} .
$$

The one-loop Coleman-Weinberg Higgs potential receives largest contribution from the top quark, as

$$
V_{\text {eff }}=-2 N_{c} \int \frac{d^{4} q}{(2 \pi)^{4}} \log \left(q^{2} \Pi_{t_{L}} \Pi_{t_{R}}+\left|\Pi_{t_{L} t_{R}}\right|^{2}\right) \simeq-\alpha \frac{h^{2}}{f^{2}}+\beta \frac{h^{4}}{f^{4}} .
$$

The coefficients $\alpha$ and $\beta$ above are integrals over the form factors. A similar contribution to $\alpha$ arises from gauge boson loops with opposite sign (largest contribution from $\mathrm{SU}(2)_{\mathrm{L}}$ gauge bosons), parametrized as [68, 69]

$$
\alpha_{g} \simeq-c_{g} \frac{1}{16 \pi^{2}} \frac{9}{2} g^{2} g_{\rho}^{2} f^{4}
$$

where $c_{g}$ is an $\mathcal{O}(1)$ positive constant absorbing the details of the integration, $g$ is the $\mathrm{SU}(2)_{\mathrm{L}}$ gauge coupling, and $g_{\rho}$ corresponds to that of strong sector spin- 1 resonances. The gauge contribution to $\beta$ is numerically small. To calculate the top-induced contribution to $\alpha$ and $\beta$, we use certain parametrization of the momentum dependent form factors based on scaling arguments. The decay constants and the top-partner masses are parametrized as

$$
F_{i}^{L, R}=\lambda_{i}^{L, R} f, \quad m_{i}=g_{i} f,
$$

where $\lambda_{i}^{L, R}$ are dimensionless constants and $g_{i}$ denote strong couplings. In the present analysis, we keep $\left|\lambda_{i}\right| / g_{i}<1$. The strong sector coupling strengths are kept well within the perturbative limits, i.e. $1<g_{i}<2 \pi$. Regarding the integrals over the form factors in fermionic sector, the loop factors and the dimensionful variables are shown explicitly. Some group theoretic factors also emerge due to the decomposition of the $\mathrm{SO}(5)$ resonances in terms of $\mathrm{SO}(4)$ multiplets. We assume that sufficient number of resonances with coupling strengths $g_{i}$ saturate the form factors, rendering the integrals finite. As an illustration, we show one of the integrals involved in the Higgs potential, as parametrized in $[3,68,69]$

$$
\int \frac{d^{4} q}{(2 \pi)^{4}}\left(\frac{\Pi_{1,2}^{L, R}(q)}{\Pi_{0}^{L, R}(q)}\right)^{n} \simeq c_{1,2}^{(n)} \frac{1}{16 \pi^{2}}\left(\frac{\Pi_{1,2}^{L, R}(0)}{\Pi_{0}^{L, R}(0)}\right)^{n} g_{i}^{4} f^{4}, \quad n=1,2,
$$

where $c_{1,2}^{(n)}$ are $\mathcal{O}(1)$ numbers and the forms factors are displayed in appendix B. Finally we use the following phenomenological constraints to generate the allowed parameter space:

$$
\begin{aligned}
169 \mathrm{GeV} & <m_{t}<176 \mathrm{GeV}, & v & =246 \mathrm{GeV}, \\
123 \mathrm{GeV} & <m_{h}<127 \mathrm{GeV}, & 1 \mathrm{TeV}<m_{i} & =g_{i} f<2 \pi f .
\end{aligned}
$$

We present the results of our numerical analysis in figure 1 . Depending on the embedding of the top quark the value of $k_{t \bar{t} h}$ varies. Interestingly, for $\mathrm{MCHM}_{14_{\mathrm{L}}-14_{\mathrm{R}}}$ and $\mathrm{MCHM}_{14_{\mathrm{L}}-5_{\mathrm{R}}}$ 

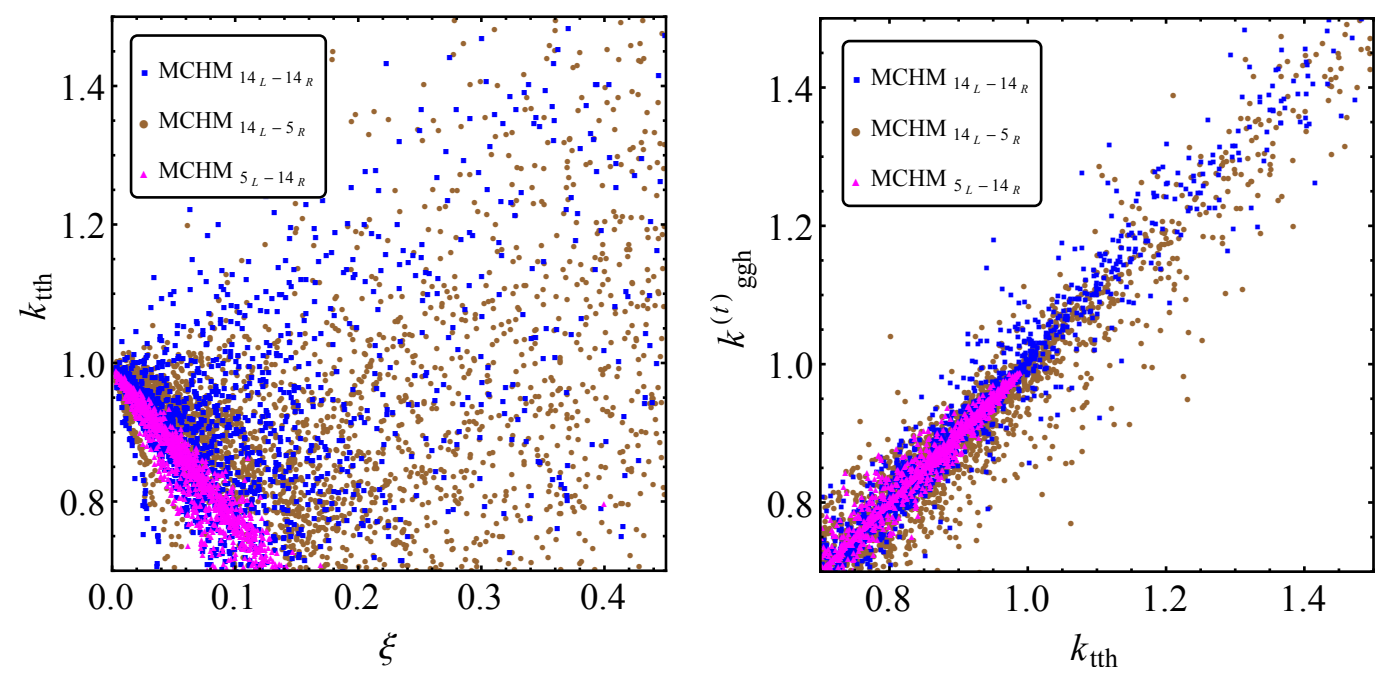

Figure 1. Results from the numerical analysis for $\operatorname{MCHM}_{14_{L}-14_{R}}$ (blue), $\operatorname{MCHM}_{14_{L}-5_{R}}$ (brown) and $\mathrm{MCHM}_{5_{\mathrm{L}}-14_{\mathrm{R}}}$ (magenta) are shown. In the left panel $k_{t \bar{t} h}$ is plotted against $\xi$ while at the right panel we show the correlation between $k_{g g h}^{(t)}$ and $k_{t \bar{t} h}$. While generating the model points we vary the strong couplings $g_{i}$ and $g_{\rho}$ in the range $[1,2 \pi]$ and $\lambda_{i}^{L, R} / g_{i}$ within $[-1,1]$. All the points shown in the plots satisfy the phenomenological constraints given in eqs. (2.9).

we get an enhancement in top Yukawa coupling compared to its SM value $\left(k_{t \bar{t} h}>1\right)$, for a large number of model points. On the other hand, for $\mathrm{MCHM}_{5_{L}-14_{R}}$ the top Yukawa is always suppressed. This is linked to the relative sign between the coefficients of the two Yukawa invariants. In figure 1 (right panel) we show the variation of $k_{g g h}^{(t)}$ with $k_{t \bar{t} h}$, and one observes that the two quantities are almost equal for all model points. This implies that the numerical impact of the wave function renormalization of the top quark is very small.

\section{$2.2 \mathrm{SO}(6) / \mathrm{SO}(5)$ coset}

The next-to-minimal model, with $\mathrm{SO}(6) / \mathrm{SO}(5)$ coset includes a real singlet scalar $(\eta)$ along with the usual Higgs doublet. Quite a few interesting features emerge in this case, depending on whether $\eta$ acquires a vev $[21,43,44]$ or not [70-73]. In the present section we discuss the effect of the $\eta$-vev and consequently the doublet-singlet scalar mixing on the Yukawa couplings. Here we follow the convention and notation as presented in [21].

In this case the structure of the Lagrangian involving the top quark is similar to eq. (2.2), with the exception that the $\Pi$-functions are dependent both on $h$ and $\eta$, as shown in appendix $\mathrm{C}$, for different representations. Although compared to $\mathrm{SO}(5) / \mathrm{SO}(4)$ coset, more possibilities of embedding $t_{L}$ and $t_{R}$ in different $\mathrm{SO}(6)$ multiplets exist, we stick to the choices shown in appendix A.2 only. The Lagrangian, in terms of the canonically normalized quantum fields $\left(h_{n}, \eta_{n}\right)$, upon electroweak symmetry breaking, can be written as

$$
\mathcal{L} \supset m_{t} \bar{t} t+k_{t \bar{t} h_{n}}\left(\frac{m_{t}}{v}\right) h_{n} \bar{t} t+k_{t \bar{t} \eta_{n}}\left(\frac{m_{t}}{v}\right) \eta_{n} \bar{t} t .
$$

Due to the doublet-singlet mixing, the state corresponding to the observed Higgs field is

$$
h^{\prime}=\cos \theta_{\text {mix }} h_{n}-\sin \theta_{\text {mix }} \eta_{n},
$$



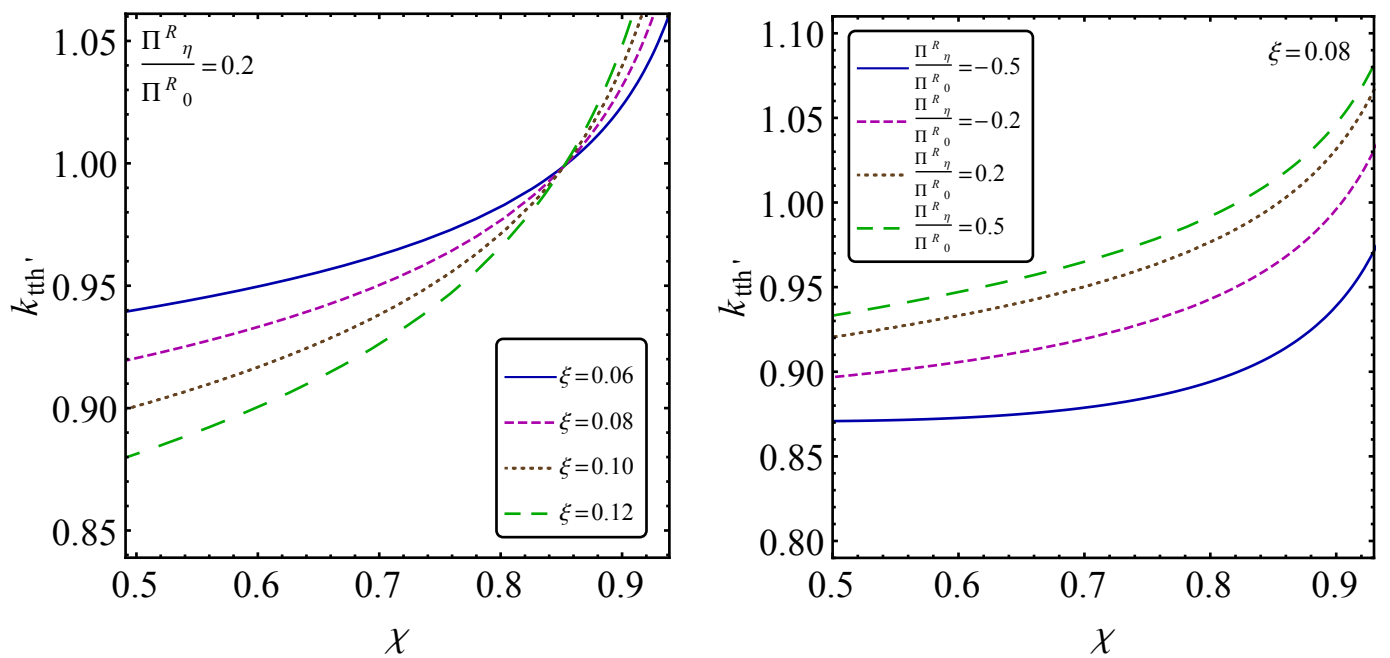

Figure 2. The variation of $k_{t \bar{t} h^{\prime}}$ with $\chi=\langle\eta\rangle^{2} / f^{2}$ is shown. In the left panel, for illustration, we keep the ratio $\Pi_{\eta}^{R} / \Pi_{0}^{R}$ (see appendix C) fixed at 0.2 , and plot the curves for different values of $\xi$. In the right panel we fix $\xi$ and plot for different values of the ratio mentioned above. While plotting the curves we assume that $\Pi_{1}^{L, R} \ll \Pi_{0}^{L, R}$ and the mixing angle $\theta_{\text {mix }}<0.25$ is respected.

where $\theta_{\text {mix }}$ denotes the amount of mixing and is constrained by the LHC Higgs data. For the case, where both $m_{\eta} \gg m_{h}$ and $\langle\eta\rangle \gg\langle h\rangle$, the mixing angle can be simply parametrized as $[44]$

$$
\theta_{\text {mix }} \sim \frac{\langle h\rangle\langle\eta\rangle}{m_{\eta}^{2}} \ll 1
$$

We also observe that

$$
k_{t \bar{t} \eta_{n}} \propto-\sqrt{\frac{\xi \chi}{1-\chi}},
$$

where $\chi=\langle\eta\rangle^{2} / f^{2}$. Because of an inherent $\mathcal{Z}_{2}$ symmetry associated with our choice of embedding, $\eta$ couples with the top quark as $\eta^{2}$. When the $\mathcal{Z}_{2}$ symmetry is spontaneously broken the dependence on $\chi$ appears. The appearance of $\xi$ is a consequence of constructing an $\mathrm{SU}(2)$ invariant Yukawa-like term involving the $\eta$ field. Finally the expression for the Yukawa coupling modifier involving the observed Higgs is obtained as

$$
k_{t \bar{t} h^{\prime}}=\cos \theta_{\mathrm{mix}} k_{t \bar{t} h_{n}}-\sin \theta_{\mathrm{mix}} k_{t \bar{t} \eta_{n}} .
$$

We show some representative plots illustrating the impact of $\chi$ on the Yukawa coupling modifier. In figure 2 we present the variation of $k_{t \bar{t} h^{\prime}}$ with $\chi$ for $\mathrm{NMCHM}_{6_{L}-6_{R}}$. Obviously extra model dependence appears in the case of symmetric representation $(\mathbf{2 0})$, where more than one Yukawa invariant exist.

\section{Effective phenomenological Lagrangian}

The modifications in the Higgs couplings as discussed in the previous section have two generic features: $(i)$ modification in $V V h$ coupling, arising from the non-linearity of the 
pNGBs, is universal as long as the coset belongs to $\mathrm{SO}(N) / \mathrm{SO}(N-1)$ group (modulo the mixing with other states), and (ii) modification of the Yukawa couplings depends on the choice of fermion embeddings. These can be captured in an effective Lagrangian as,

$$
\begin{aligned}
\mathcal{L}= & \mathcal{L}_{\text {SMEFT }}+\Delta \mathcal{L} \\
\mathcal{L}_{\text {SMEFT }} \supset & \partial_{\mu} H^{\dagger} \partial^{\mu} H+\frac{g^{2}}{2} H^{\dagger} H\left(W_{\mu} W^{\mu}+\frac{1}{2 \cos \theta_{w}^{2}} Z_{\mu} Z^{\mu}\right)-\sum_{u} y_{u} \bar{q}_{L} H^{c} u_{R}-\sum_{d} y_{d} \bar{q}_{L} H d_{R} \\
& -\sum_{i=u, d} \frac{\alpha_{s}}{8 \pi} y_{i} b_{i}^{s} \frac{H^{\dagger} H}{v^{2}} G_{\mu \nu} G^{\mu \nu}-\frac{\alpha_{e m}}{8 \pi}\left(\sum_{i=u, d} y_{i} b_{i}^{e m}-g b_{W}^{e m}\right) \frac{H^{\dagger} H}{v^{2}} F_{\mu \nu} F^{\mu \nu} .
\end{aligned}
$$

Above, $\mathcal{L}_{\text {SMEFT }}$ comprises of the Standard Model effective Lagrangian with relevant dimension- 4 and dimension- 6 operators, where the explicit forms of the numerical coefficients $b_{i}$ are given in [74]. In the $\mathrm{SO}(5) / \mathrm{SO}(4)$ models, additional contributions to dimension- 6 operators emerge, given by

$$
\begin{aligned}
\Delta \mathcal{L} \supset & \frac{1}{2 f^{2}} \partial_{\mu}\left(H^{\dagger} H\right) \partial^{\mu}\left(H^{\dagger} H\right)-\sum_{u}\left(\Delta_{u}^{\prime}+\delta_{u}\right) y_{u} \frac{H^{\dagger} H}{f^{2}} \bar{q}_{L} H^{c} u_{R}-\sum_{d}\left(\Delta_{d}^{\prime}+\delta_{d}\right) y_{d} \frac{H^{\dagger} H}{f^{2}} \bar{q}_{L} H d_{R} \\
& -\sum_{i=u, d} \frac{\alpha_{s}}{8 \pi} \Delta_{i}^{\prime} y_{i} b_{i}^{s} \frac{H^{\dagger} H}{f^{2}} G_{\mu \nu} G^{\mu \nu}-\frac{\alpha_{e m}}{8 \pi} \sum_{i=u, d} \Delta_{i}^{\prime} y_{i} b_{i}^{e m} \frac{H^{\dagger} H}{f^{2}} F_{\mu \nu} F^{\mu \nu} .
\end{aligned}
$$

In the above Lagrangian we have dropped terms which are highly constrained by the electroweak precision observables [45]. One can read off the Yukawa and $g g h / \gamma \gamma h$ coupling modifiers as

$k_{f \bar{f} h}=1+\left(\Delta_{f}^{\prime}+\delta_{f}-\frac{1}{2}\right) \xi \equiv 1+\left(\Delta_{f}+\delta_{f}\right) \xi, \quad k_{g g h / \gamma \gamma h}^{(f)}=1+\left(\Delta_{f}^{\prime}-\frac{1}{2}\right) \xi \equiv 1+\Delta_{f} \xi$,

and the modifier for $V V h$ coupling as

$$
k_{V V h}=1-\frac{1}{2} \xi
$$

While $\xi$ represents the ratio of the weak scale to the effective scale of the theory, thus naturally controlling the coupling modifiers, a brief discussion of the other two parameters, namely $\Delta$ and $\delta$, in the effective Lagrangian is in order. The origin of $\Delta$ can be traced back to the nonlinear realization of the pNGB sector. In scenarios containing only one Yukawa invariant this is a numerical constant (e.g. in $\mathrm{MCHM}_{5_{L}-5_{R}}, \Delta \simeq-3 / 2$ ), while in the extended models with several invariants this factor may deviate depending on the details of the strong sector resonances. In contrast, $\delta$ reflects the effect of partial composite nature of the top quark in these theories, contributing to the anomalous dimension of the top quark. In the effective $g g h$ and $\gamma \gamma h$ vertex, in fact, contributions from the wave function renormalization cancel against the resonance loop contributions [31, 67]. In our phenomenological analysis, that follows in the next section, we will employ the effective Lagrangian (eqs. (3.1) and (3.2)), to confront the LHC Higgs data. All fitting are done assuming $\Delta_{t}$ and $\delta_{t}$ to be free parameters. Further we assume that the bottom and $\tau$ 


\begin{tabular}{|c|c|}
\hline Modifiers & Dependence on parameters \\
\hline$k_{V V h}(V V=W W, Z Z)$ & $1-\frac{1}{2} \xi$ \\
$k_{t \bar{t} h}$ & $1+\left(\Delta_{t}+\delta_{t}\right) \xi$ \\
$k_{g g h / \gamma \gamma h}^{(t)}$ & $1+\Delta_{t} \xi$ \\
$k_{b \bar{b} h}$ & $1-\frac{3}{2} \xi$ \\
$k_{g g h}^{(b)}$ & $1-\frac{3}{2} \xi$ \\
$k_{\tau \bar{\tau} h}$ & $1-\frac{3}{2} \xi$ \\
\hline
\end{tabular}

Table 2. Scaling of the Higgs effective couplings for $\mathrm{SO}(5) / \mathrm{SO}(4)$ model.

Yukawa couplings are modified only by the universal factor $\Delta_{b}=\Delta_{\tau}=-3 / 2$, i.e. they are always suppressed compared to their SM values. We also make a reasonable approximation $\delta_{b}=\delta_{\tau}=0$. A complete list of all the coupling modifiers within the $\mathrm{SO}(5) / \mathrm{SO}(4)$ model is given in table $2 .^{3}$ The explicit expressions of $\Delta_{t}$ and $\delta_{t}$ in terms of the form factors are defined in table 5 of appendix D.

The main feature that gets added when one moves to the next-to-minimal model is the presence of an additional singlet scalar and its mixing with the Higgs doublet. A description of the composite models including a SM singlet in the context of a strongly interacting light Higgs can be found in [75]. Here we present a simplified effective Lagrangian keeping only the dominant terms. We add the following piece involving $\eta$ to eqs. (3.1) and (3.2),

$$
\Delta \mathcal{L}_{\eta} \sim \frac{1}{2} \partial_{\mu} \eta \partial^{\mu} \eta-\sum_{u} y_{u}\left(\Delta_{u}^{\eta}\right)^{\prime} \frac{\eta^{2}}{f^{2}} \bar{q}_{L} H^{c} u_{R}-\sum_{d} y_{d}\left(\Delta_{d}^{\eta}\right)^{\prime} \frac{\eta^{2}}{f^{2}} \bar{q}_{L} H d_{R}
$$

Note that the dimension-5 operators involving a single $\eta$ field is not allowed in the presence of a $\mathcal{Z}_{2}$ symmetry, as discussed in previous section. Due to doublet-singlet scalar mixing $\left(\theta_{\text {mix }}\right)$, the Yukawa modifier for the observed Higgs boson $\left(h^{\prime}\right)$ assumes the following form

$$
k_{f \bar{f} h^{\prime}}=\cos \theta_{\text {mix }}\left(1+\left(\Delta_{f}+\delta_{f}\right) \xi\right)+\sin \theta_{\text {mix }} \Delta_{f}^{\eta} \sqrt{\xi},
$$

where $\Delta_{f}^{\eta}$ is a function of $\left(\Delta_{f}^{\eta}\right)^{\prime}$ and the $\eta$-vev. The expressions for $\Delta_{t}^{\eta}$ for different representations are given in table 6 of appendix D. In the following analysis we assume $\Delta_{t}^{\eta}=\Delta_{b}^{\eta}$ for simplicity.

The $V V h^{\prime}$ coupling modifier now picks up the additional factor $\cos \theta_{\text {mix }}$ compared to the minimal coset (see eq. (3.4))

$$
k_{V V h^{\prime}}=\cos \theta_{\text {mix }} \sqrt{1-\xi} .
$$

\footnotetext{
${ }^{3}$ In [53], effective $g g h$ and $\gamma \gamma h$ coupling modifiers have been calculated keeping only the dominant terms: $k_{g g h} \simeq 1.06\left(k_{g g h}^{(t)}\right)^{2}+0.01\left(k_{g g h}^{(b)}\right)^{2}-0.07 k_{g g h}^{(b)} k_{g g h}^{(t)}$ and $k_{\gamma \gamma h} \simeq 0.07\left(k_{\gamma \gamma h}^{(t)}\right)^{2}+1.59 k_{W W h}^{2}-0.66 k_{\gamma \gamma h}^{(t)} k_{W W h}$.
} 


\section{Constraints from LHC Run 1 and Run 2 Higgs data}

In this section we discuss how the Higgs coupling modifications confront the recent LHC data [53-66]. We perform a $\chi^{2}$ fit to assess the present constraints starting from the effective Lagrangian introduced in the previous section. We use the combined ATLAS+CMS Run 1 results for signal strengths, given by the 'six-parameter' fit as shown in table 15 of [53]. The so far available Run $2(13 \mathrm{TeV})$ results are summarized in table 3.

The effective Lagrangian given in eq. (3.2), which corresponds to $\mathrm{SO}(5) / \mathrm{SO}(4)$ coset, have three independent parameters $\xi, \Delta_{t}$ and $\delta_{t}$. Using this parametrization we calculate the Higgs signal strengths in various final states normalized to their SM values. These are then compared with the data using the $\chi^{2}$ fit. The minimum value of the $\chi^{2}$ and corresponding best-fit values of the parameters in the extended models are given below.

- Run 1:

$$
\chi_{\min }^{2}=1.92, \quad \Delta_{t}=-0.31, \quad \delta_{t}=0.10, \quad \xi=0.13,
$$

- Run $1+$ Run 2 :

$$
\chi_{\min }^{2}=18.85, \quad \Delta_{t}=-0.06, \quad \delta_{t}=0.02, \quad \xi=0.05 .
$$

This may be compared with a similar fit obtained for the $\mathrm{MCHM}_{5_{L}-5_{R}}$ with only one free parameter $\xi$. The best-fit values are given by

- Run 1:

$$
\chi_{\min }^{2}=3.43, \quad \xi=0.007,
$$

- Run $1+$ Run 2 :

$$
\chi_{\min }^{2}=19.72, \quad \xi=0.00 .
$$

In figures 3 we plot $\Delta \chi^{2}$ as a function of $\xi$, corresponding to our effective Lagrangian eq. (3.2), with all other parameters fixed to their best-fit values. For comparison, we also show the curve for $\mathrm{MCHM}_{5_{\mathrm{L}}-5_{\mathrm{R}}}$, and our results agree with [44] wherever we overlap. For the extended models, we obtain a lower bound $f \geq 465 \mathrm{GeV}$ at $95 \%$ CL from Run 1 data only. This should be compared with $f \geq 780 \mathrm{GeV}$ at $95 \%$ CL for $\mathrm{MCHM}_{5_{\mathrm{L}}-5_{\mathrm{R}}}$. The relaxation of the bounds on $f$ for the extended models follows from the reduced correlation between $k_{t \bar{t} h}$ and $k_{V V h}$ in the effective Lagrangian in eq. (3.2) as compared to the tight correlation in $\mathrm{MCHM}_{5_{\mathrm{L}}-5_{\mathrm{R}}}$. We find that combined Run 1 and Run 2 data give significantly more stringent lower bound, namely, $f \geq 640 \mathrm{GeV}$ at $95 \%$ CL for the extended models.

In figures 4 we check whether the extended models fit the data better (i.e. $\chi^{2} /$ d.o.f. is lower) than $\mathrm{MCHM}_{5_{\mathrm{L}}-5_{R}}$. In the blue shaded region the extended models, as parametrically encoded in the effective Lagrangian in eq. (3.2), fit relatively better for the entire range of $\xi$. On the same plot we also throw the actual model points, with the resonance masses and decay constants as the strong sector inputs, discussed on a case-by-case basis in section 2.1, satisfying the constraints shown in eq. (2.9). In figures 5, the experimentally preferred regions for the coupling modifiers are shown at $68 \%$ and $95 \%$ CL in the $\left(k_{t \bar{t}}-\xi\right)$ and 


\begin{tabular}{|c|c|c|c|c|}
\hline \multicolumn{5}{|c|}{ Run 2 Data } \\
\hline Collaboration & References & Decay Channels & Production Modes & Results \\
\hline \multirow{10}{*}{ ATLAS } & {$[54]$} & $W W$ & $V B F$ & $1.70_{-0.90}^{+1.10}$ \\
\hline & \multirow{4}{*}[55,56]{} & \multirow{4}{*}{$\gamma \gamma$} & $g g F$ & $0.80_{-0.18}^{+0.19}$ \\
\hline & & & $V B F$ & $2.10_{-0.60}^{+0.60}$ \\
\hline & & & $V H$ & $0.70_{-0.80}^{+0.90}$ \\
\hline & & & $t \bar{t} H$ & $0.60_{-0.60}^{+0.70}$ \\
\hline & \multirow[t]{2}{*}[57]{} & \multirow[t]{2}{*}{$Z Z^{*}$} & $g g F$ & $1.11_{-0.22}^{+0.25}$ \\
\hline & & & $V B F$ & $4.00_{-1.46}^{+1.77}$ \\
\hline & \multirow[t]{2}{*}[56,58]{} & \multirow[t]{2}{*}{$b \bar{b}$} & $V H$ & $1.20_{-0.36}^{+0.42}$ \\
\hline & & & $t \bar{t} H$ & $0.80_{-0.60}^{+0.60}$ \\
\hline & {$[56,59]$} & Multileptons & $t \bar{t} H$ & $1.60_{-0.40}^{+0.50}$ \\
\hline \multirow{10}{*}{ CMS } & {$[60]$} & $W W$ & $g g F+V B F+V H$ & $1.050_{-0.26}^{+0.26}$ \\
\hline & \multirow{4}{*}[61]{} & \multirow{4}{*}{$\gamma \gamma$} & $g g F$ & $1.11_{-0.18}^{+0.19}$ \\
\hline & & & $V B F$ & $0.50_{-0.50}^{+0.60}$ \\
\hline & & & $V H$ & $2.30_{-1.00}^{+1.10}$ \\
\hline & & & $t \bar{t} H$ & $2.20_{-0.80}^{+0.90}$ \\
\hline & {$[62]$} & $Z Z^{*}$ & $g g F+t \bar{t} H$ & $1.20_{-0.31}^{+0.35}$ \\
\hline & [63] & $\tau \tau$ & $g g F+V B F+V H$ & $1.06_{-0.24}^{+0.25}$ \\
\hline & {$[64]$} & $b \bar{b}$ & $Z H$ & $1.20_{-0.40}^{+0.40}$ \\
\hline & {$[65]$} & $\tau_{h}+$ others & $t \bar{t} H$ & $0.72_{-0.53}^{+0.62}$ \\
\hline & {$[66]$} & Multileptons & $t \bar{t} H$ & $1.50_{-0.50}^{+0.50}$ \\
\hline
\end{tabular}

Table 3. Results from the ATLAS and CMS collaborations for Higgs signal strengths at $13 \mathrm{TeV}$ are tabulated.

$\left(k_{g g h}^{(t)}-k_{t \bar{t} h}\right)$ planes. The model points are observed to span over a large range of the preferred regions. It may be noted that present experimental precision is not sensitive to the value of $\delta_{t}$ separately; what is in fact bounded is the combination $\left(\Delta_{t}+\delta_{t}\right)$. Future colliders may have sufficient precision to sense the different modifications in the top Yukawa coupling and the effective $g g h$ coupling.

Moving to the next-to-minimal coset, we deal with a new feature that the Higgs doublet now mixes with a real singlet. The mixing results in a further suppression of the observed Higgs boson coupling to the massive gauge bosons. The Yukawa couplings are modified too because of the presence of a singlet. We perform a similar $\chi^{2}$ analysis with the combined Run 1 and Run 2 results to impose an upper bound on the amount of mixing. Figure 6 shows that the maximum amount of mixing allowed so far at $95 \% \mathrm{CL}$ is $\theta_{\text {mix }} \sim 0.35$. Future data would constrain it even further [21]. 

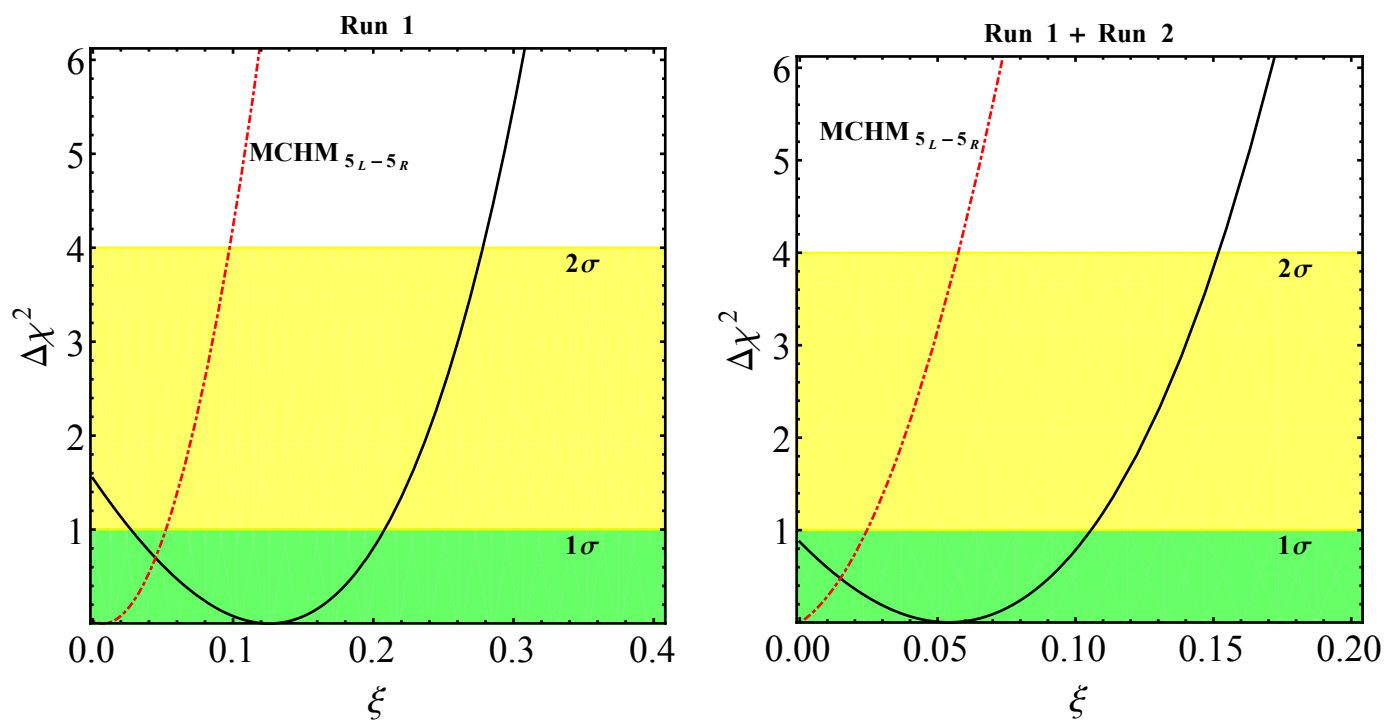

Figure 3. Results of $\chi^{2}$ analysis for Run 1 and Run $1+$ Run 2 datasets are shown in the left and right panels, respectively. Solid black line represents $\Delta \chi^{2}=\chi^{2}-\chi_{\min }^{2}$ for the extended models, while the red dashed lines represents the same for $\mathrm{MCHM}_{5_{L}-5_{R}}$. Green and yellow regions denote the allowed range for $\xi$ at $68 \%$ and $95 \%$ CL, respectively.
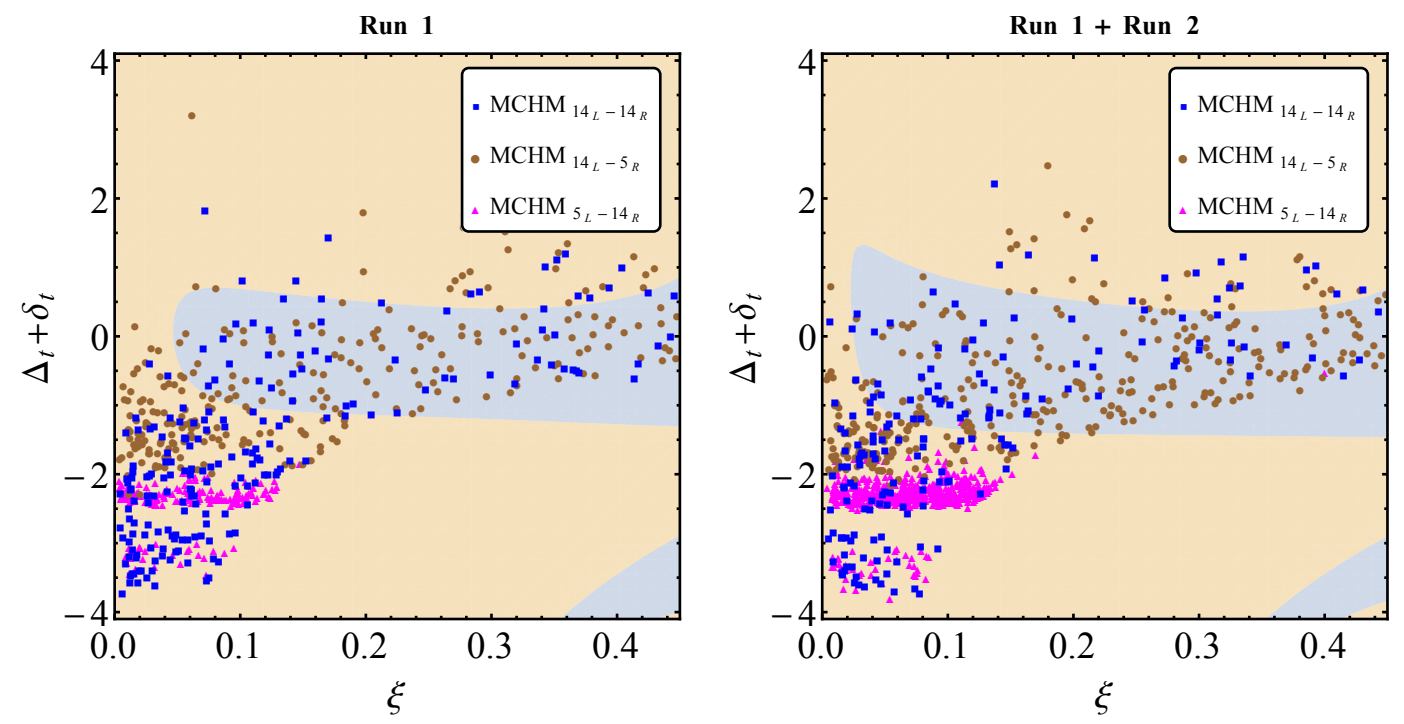

Figure 4. In both the left and right panels, blue shaded regions denote relatively lower values of $\chi^{2} /$ d.o.f. for the extended models, given by the effective Lagrangian in eq. (3.2), compared to the $\mathrm{MCHM}_{5_{\mathrm{L}}-5_{\mathrm{R}}}$. Model points, with resonance masses and decay constants as inputs, satisfying the constraints of eq. (2.9), are superimposed.

\section{Conclusions}

Non-linearity of pNGB dynamics modifies the Higgs boson couplings with the weak gauge bosons as well as with the fermions compared to their SM expectations. The ratio $\xi$, which parametrizes the hierarchy between the weak scale and the strong sector spontaneous 

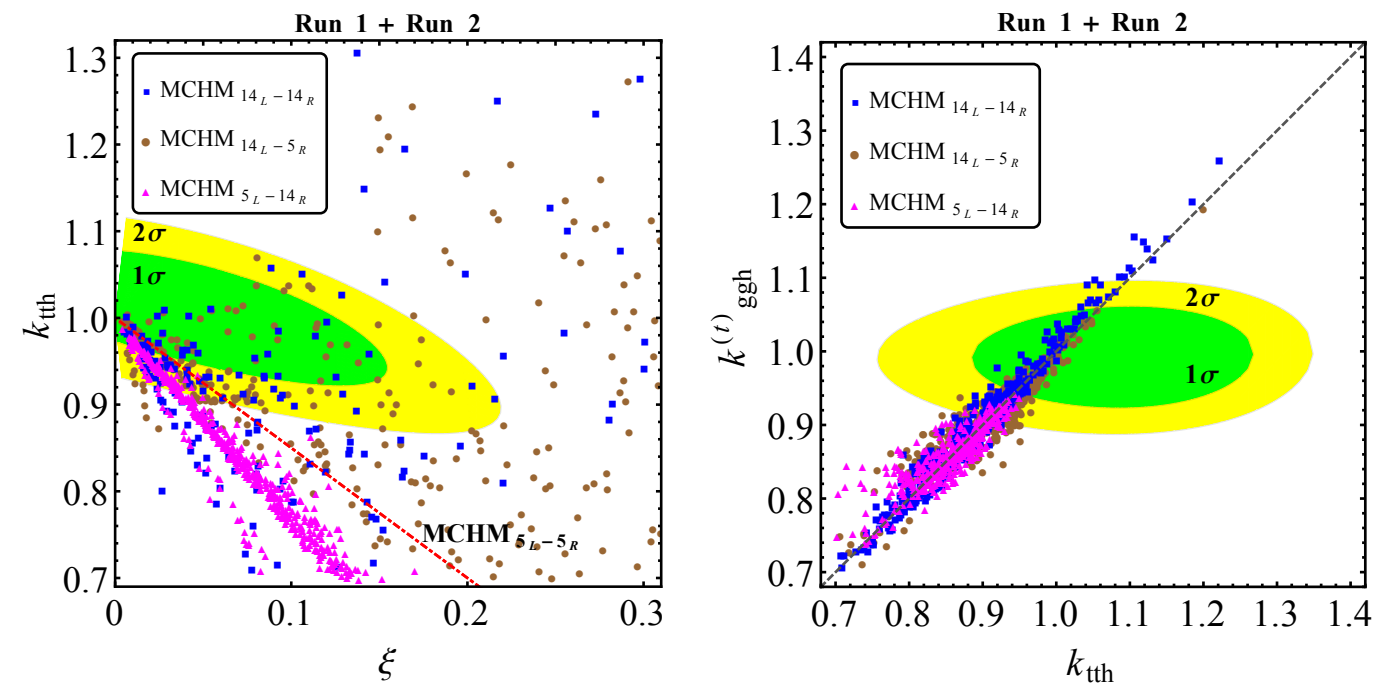

Figure 5. We present the regions in the $k_{t \bar{t} h}-\xi$ and $k_{g g h}^{(t)}-k_{t \bar{t} h}$ planes allowed at $68 \%$ (green) and $95 \%$ (yellow) CL using combined Run 1 and available Run 2 data. In the left panel the red line corresponds to $\mathrm{MCHM}_{5_{\mathrm{L}}-5_{\mathrm{R}}}$. On the right panel, the grey dashed line corresponds to $\delta_{t}=0$. Valid 'extended model' points are observed to lie within the experimentally allowed regions.

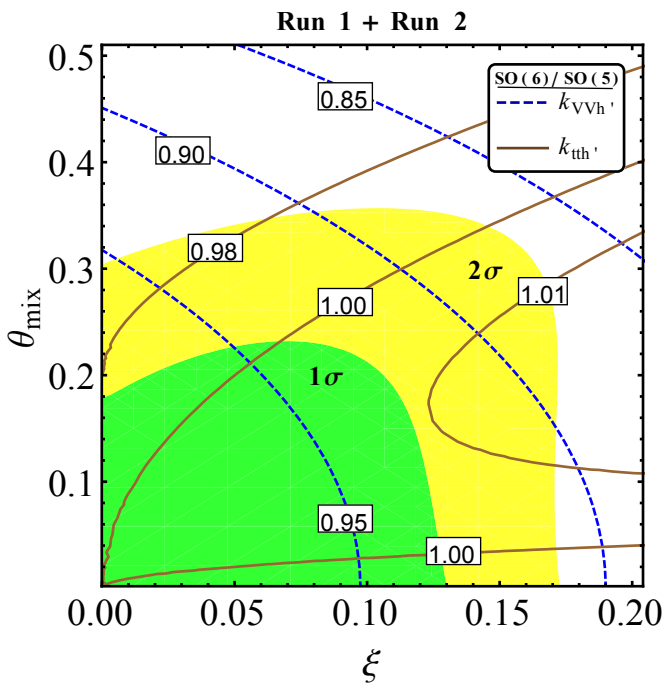

Figure 6. For the next-to-minimal model, the allowed regions in the $\theta_{\text {mix }}-\xi$ plane at $68 \%$ (green) and $95 \%$ (yellow) CL are shown using the combined Run 1 and available Run 2 data. The brown lines represent the contours of fixed $k_{t \bar{t} h^{\prime}}$, while the blue dashed lines correspond to that of $k_{V V h^{\prime}}$.

symmetry breaking scale, controls this deformation. In $\mathrm{MCHM}_{5_{\mathrm{L}}-5_{R}}$, the Yukawa sector contains a single invariant. Here, the single parameter $\xi$ appears in the modifications of both $V V h$ and $f \bar{f} h$ couplings, leading to a rather strong lower limit $f \geq 1 \mathrm{TeV}$, as the data show increasing affinity towards the SM predictions. In the extended models, $\mathrm{MCHM}_{14_{\mathrm{L}}-14_{\mathrm{R}}}, \mathrm{MCHM}_{14_{\mathrm{L}}-5_{\mathrm{R}}}$ and $\mathrm{MCHM}_{5_{\mathrm{L}}-14_{\mathrm{R}}}$, owing to the presence of more than one invariant in the Yukawa sector, the $f \bar{f} h$ coupling modifier depends on other parameters of 
the strong sector in addition to $\xi$. This releases the tension leading to a new lower limit $f \geq 640 \mathrm{GeV}$, which is much relaxed compared to the limit in $\mathrm{MCHM}_{5_{\mathrm{L}}-5_{\mathrm{R}}}$.

An important feature of these models is the emergence of a parametric difference in the top Yukawa and the effective gluon-gluon-Higgs vertices. This arises because of a cancellation between the top-partner resonance masses in the loop with the wave function renormalization of the top quark in the calculation of the effective $g g h$ vertex. However, the present data is insensitive to smell this difference.

We have in fact constructed a phenomenological Lagrangian which captures the effects of a vast array of such models with different fermionic representations. We have constrained the parameters of this Lagrangian using LHC data and observed that the allowed regions are quite consistent with a reasonable choice of strong sector input parameters of the individual models which yield correct values of $m_{t}, v$ and $m_{h}$.

We have extended our analysis to the next-to-minimal model as well. The appearance of a real singlet scalar adds a new twist to phenomenology, whose mixing with the Higgs doublet is constrained by the LHC data. Interestingly, the singlet scalar also contributes to the top Yukawa through an effective higher dimensional operator.

Our analysis shows that further precision, likely to be achieved in future colliders, would constrain these scenarios to the extent that individual models could be discriminated, and the proposition that the Higgs boson may have a spatial extension would be challenged with more ammunition.

\section{Acknowledgments}

We thank S. Bhattacharya, M. R. Gangopadhyay, S. Roy Chowdhury, K. Mondal, C. Mondal and D. Das for discussions. AB acknowledges financial support from Department of Atomic Energy, Government of India. GB acknowledges support of the J.C. Bose National Fellowship from the Department of Science and Technology, Government of India (SERB Grant No. SB/S2/JCB-062/2016). Support from the Indo French Center for Promotion of Advanced Research (CEFIPRA Project No. 5404-2) is also acknowledged by GB and NK. TSR acknowledges the hospitality provided by ICTP, Italy, under the Associate program, during the initial stages of the project. TSR is partially supported by the Department of Science and Technology, Government of India, under the Grant Agreement number IFA13-PH-74 (INSPIRE Faculty Award).

\section{A Fermion embeddings}

\section{A.1 $\mathrm{SO}(5) / \mathrm{SO}(4)$ coset}

Fundamental $\mathbf{5}$ and symmetric $\mathbf{1 4}$ representations of $\mathrm{SO}(5)$ can be decomposed under the unbroken $\mathrm{SO}(4) \equiv \mathrm{SU}(2)_{\mathrm{L}} \times \mathrm{SU}(2)_{\mathrm{R}}$ as follows:

$$
\begin{aligned}
\mathbf{5} & =\mathbf{1} \oplus \mathbf{4}=(\mathbf{1}, \mathbf{1}) \oplus(\mathbf{2}, \mathbf{2}), \\
\mathbf{1 4} & =\mathbf{1} \oplus \mathbf{4} \oplus \mathbf{9}=(\mathbf{1}, \mathbf{1}) \oplus(\mathbf{2}, \mathbf{2}) \oplus(\mathbf{3}, \mathbf{3}) .
\end{aligned}
$$


We embed $t_{L}$ into the $(\mathbf{2}, \mathbf{2})$ 's so that the correction to $Z b \bar{b}$ vertex is under control, while $t_{R}$ is embedded into the $(\mathbf{1}, \mathbf{1})$. The embeddings of the top quarks into incomplete multiplets of 5 and 14 are given below.

$$
Q_{t_{L}}^{5}=\left(\Psi_{(2,2)}, 0\right)^{T}, \quad T_{t_{R}}^{5}=\left(0,0,0,0, t_{R}\right)^{T},
$$

and

$$
Q_{t_{L}}^{14}=\left(\begin{array}{c|c}
0_{4 \times 4} & \frac{\Psi_{(2,2)}^{T}}{\sqrt{2}} \\
\hline \frac{\Psi_{(2,2)}}{\sqrt{2}} & 0
\end{array}\right), \quad T_{t_{R}}^{14}=\left(\begin{array}{cc|c}
-\frac{t_{R}}{2 \sqrt{5}} I_{4} & 0_{4 \times 1} \\
\hline 0_{1 \times 4} & 4 \frac{t_{R}}{2 \sqrt{5}}
\end{array}\right)
$$

where

$$
\Psi_{(2,2)}=\frac{1}{\sqrt{2}}\left(i b_{L}, b_{L}, i t_{L},-t_{L}\right)
$$

\section{A.2 $\mathrm{SO}(6) / \mathrm{SO}(5)$ coset}

Decomposition of different representations of $\mathrm{SO}(6)$, used in the main text, under the maximal subgroup $\mathrm{SO}(6) \supset \mathrm{SO}(4) \times \mathrm{SO}(2) \simeq \mathrm{SU}(2)_{L} \times \mathrm{SU}(2)_{R} \times \mathrm{U}(1)_{\eta}$, is as follows:

$$
\begin{aligned}
\mathbf{6}_{0} & =(\mathbf{2}, \mathbf{2})_{0} \oplus(\mathbf{1}, \mathbf{1})_{2} \oplus(\mathbf{1}, \mathbf{1})_{-2}, \\
\mathbf{1 5}_{0} & =(\mathbf{1}, \mathbf{1})_{0} \oplus(\mathbf{2}, \mathbf{2})_{2} \oplus(\mathbf{2}, \mathbf{2})_{-2} \oplus(\mathbf{3}, \mathbf{1})_{0} \oplus(\mathbf{1}, \mathbf{3})_{0}, \\
\mathbf{2 0}_{0} & =(\mathbf{1}, \mathbf{1})_{0} \oplus(\mathbf{1}, \mathbf{1})_{4} \oplus(\mathbf{1}, \mathbf{1})_{-4} \oplus(\mathbf{2}, \mathbf{2})_{2} \oplus(\mathbf{2}, \mathbf{2})_{-2} \oplus(\mathbf{3}, \mathbf{3})_{0},
\end{aligned}
$$

where the subscripts denote the charges under $\mathrm{U}(1)_{\eta}$. Embedding of $t_{L}$ and $t_{R}$ in the above representations are given as

$$
\begin{aligned}
& Q_{t_{L}}^{6}=\left(\Psi_{(2,2)}, 0,0\right)^{T}, \quad T_{t_{R}}^{6}=\left(0,0,0,0,0, t_{R}\right)^{T},
\end{aligned}
$$

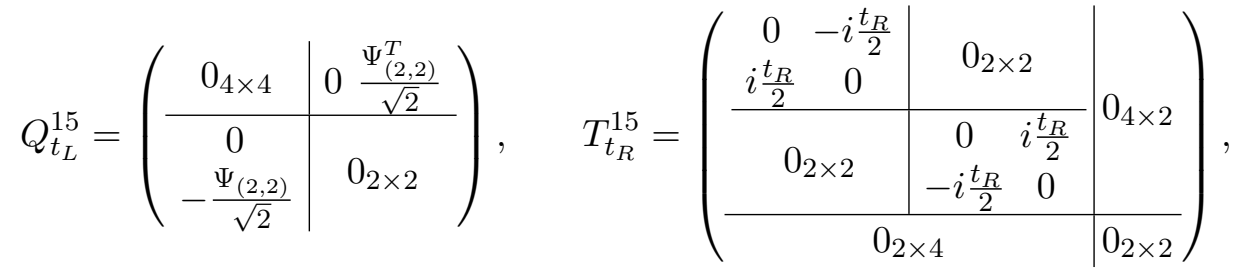

and

$$
Q_{t_{L}}^{20}=\left(\begin{array}{c|c}
0_{4 \times 4} & 0 \frac{\Psi_{(2,2)}^{T}}{\sqrt{2}} \\
\hline 0 & 0_{2 \times 2}
\end{array}\right), \quad T_{t_{R}}^{20}=\left(\begin{array}{cc|c}
-\frac{t_{R}}{2 \sqrt{3}} I_{4} & 0_{4 \times 2} \\
\hline 0_{2 \times 4} & \frac{t_{R}}{\sqrt{3}} I_{2}
\end{array}\right)
$$

\section{B Details of form factors}

The form factors appearing in table 1 can be decomposed under unbroken $\mathrm{SO}(4)$ and written in terms of masses and decay constants of the resonances. Detailed expressions

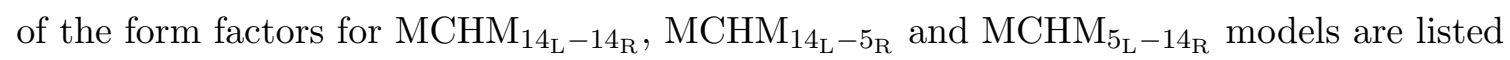
below (for explicit calculations see [31]). 
$\mathrm{MCHM}_{14_{\mathrm{L}}-14_{\mathrm{R}}}$

$$
\left.\begin{array}{rl}
\Pi_{0}^{L} & =1+\frac{\left|F_{4}^{L}\right|^{2}}{q^{2}+m_{4}^{2}}, \\
\Pi_{1}^{L} & =\left(\frac{5}{4} \frac{\left|F_{1}^{L}\right|^{2}}{q^{2}+m_{1}^{2}}-\frac{5}{2} \frac{\left|F_{4}^{L}\right|^{2}}{q^{2}+m_{4}^{2}}+\frac{5}{4} \frac{\left|F_{9}^{L}\right|^{2}}{q^{2}+m_{9}^{2}}\right), \\
\Pi_{2}^{L} & =\left(-\frac{5}{4} \frac{\left|F_{1}^{L}\right|^{2}}{q^{2}+m_{1}^{2}}+2 \frac{\left|F_{4}^{L}\right|^{2}}{q^{2}+m_{4}^{2}}-\frac{3}{4} \frac{\left|F_{9}^{L}\right|^{2}}{q^{2}+m_{9}^{2}}\right), \\
\Pi_{0}^{R} & =1+\frac{\left|F_{1}^{R}\right|^{2}}{q^{2}+m_{1}^{2}}, \\
\Pi_{1}^{R} & =\left(\frac{5}{2} \frac{\left|F_{4}^{R}\right|^{2}}{q^{2}+m_{4}^{2}}-\frac{5}{2} \frac{\left|F_{1}^{R}\right|^{2}}{q^{2}+m_{1}^{2}}\right), \\
\Pi_{2}^{R} & =\left(\frac{25}{16} \frac{\left|F_{1}^{R}\right|^{2}}{q^{2}+m_{1}^{2}}-\frac{5}{2} \frac{\left|F_{4}^{R}\right|^{2}}{q^{2}+m_{4}^{2}}+\frac{15}{16} \frac{\left|F_{9}^{R}\right|^{2}}{q^{2}+m_{9}^{2}}\right), \\
\Pi_{1}^{L R} & =-\frac{\sqrt{5}}{2}\left(\frac{F_{1}^{L} F_{1}^{R *} m_{1}}{q^{2}+m_{1}^{2}}-\frac{F_{4}^{L} F_{4}^{R *} m_{4}}{q^{2}+m_{4}^{2}}\right), \\
\Pi_{2}^{L R} & =-\left(-\frac{5 \sqrt{5}}{8} \frac{F_{1}^{L} F_{1}^{R *} m_{1}}{q^{2}+m_{1}^{2}}+\sqrt{5} \frac{F_{4}^{L} F_{4}^{R *} m_{4}}{q^{2}+m_{4}^{2}}-\frac{3 \sqrt{5}}{8} \frac{F_{9}^{L} F_{9}^{R *} m_{9}}{q^{2}+m_{9}^{2}}\right) .
\end{array}\right\}
$$

$\operatorname{MCHM}_{14_{L}-5_{R}}$

$$
\begin{aligned}
\Pi_{0}^{L} & =1+\frac{\left|F_{4}^{L}\right|^{2}}{q^{2}+m_{4}^{2}}, \\
\Pi_{1}^{L} & =\frac{5}{4} \frac{\left|F_{1}^{L}\right|^{2}}{q^{2}+m_{1}^{2}}-\frac{5}{2} \frac{\left|F_{4}^{L}\right|^{2}}{q^{2}+m_{4}^{2}}+\frac{5}{4} \frac{\left|F_{9}^{L}\right|^{2}}{q^{2}+m_{4}^{2}}, \\
\Pi_{2}^{L} & =2 \frac{\left|F_{4}^{L}\right|^{2}}{q^{2}+m_{4}^{2}}-\frac{5}{4} \frac{\left|F_{1}^{L}\right|^{2}}{q^{2}+m_{1}^{2}}-\frac{3}{4} \frac{\left|F_{9}^{L}\right|^{2}}{q^{2}+m_{4}^{2}} \\
\Pi_{0}^{R} & =1+\frac{\left|F_{1}^{R}\right|^{2}}{q^{2}+m_{1}^{2}}, \\
\Pi_{1}^{R} & =\frac{\left|F_{4}^{R}\right|^{2}}{q^{2}+m_{4}^{2}}-\frac{\left|F_{1}^{R}\right|^{2}}{q^{2}+m_{1}^{2}}, \\
\Pi_{1}^{L R} & =\frac{1}{\sqrt{2}} \frac{F_{4}^{L} F_{4}^{R *} m_{4}}{q^{2}+m_{4}^{2}}-\frac{\sqrt{5}}{2} \frac{F_{1}^{L} F_{1}^{R *} m_{1}}{q^{2}+m_{1}^{2}} \\
\Pi_{2}^{L R} & =\frac{\sqrt{5}}{2} \frac{F_{1}^{L} F_{1}^{R *} m_{1}}{q^{2}+m_{1}^{2}}-\sqrt{2} \frac{F_{4}^{L} F_{4}^{R *} m_{4}}{q^{2}+m_{4}^{2}} .
\end{aligned}
$$

$\mathrm{MCHM}_{5_{L}-14_{R}}$

$$
\left.\begin{array}{rl}
\Pi_{0}^{L} & =1+\frac{\left|F_{4}^{L}\right|^{2}}{q^{2}+m_{4}^{2}} \\
\Pi_{1}^{L} & =\frac{1}{2}\left(\frac{\left|F_{1}^{L}\right|^{2}}{q^{2}+m_{1}^{2}}-\frac{\left|F_{4}^{L}\right|^{2}}{q^{2}+m_{4}^{2}}\right) \\
\Pi_{0}^{R} & =1+\frac{\left|F_{1}^{R}\right|^{2}}{q^{2}+m_{1}^{2}} \\
\Pi_{1}^{R} & =\frac{5}{2}\left(\frac{\left|F_{4}^{R}\right|^{2}}{q^{2}+m_{4}^{2}}-\frac{\left|F_{1}^{R}\right|^{2}}{q^{2}+m_{1}^{2}}\right) \\
\Pi_{2}^{R} & =\frac{25}{16} \frac{\left|F_{1}^{R}\right|^{2}}{q^{2}+m_{1}^{2}}-\frac{5}{2} \frac{\left|F_{4}^{R}\right|^{2}}{q^{2}+m_{4}^{2}}+\frac{15}{16} \frac{\left|F_{9}^{R}\right|^{2}}{q^{2}+m_{4}^{2}} \\
\Pi_{1}^{L R} & =\frac{\sqrt{5}}{2} \frac{F_{4}^{L} F_{4}^{R *} m_{4}}{q^{2}+m_{4}^{2}}-\frac{1}{\sqrt{2}} \frac{F_{1}^{L} F_{1}^{R *} m_{1}}{q^{2}+m_{1}^{2}} \\
\Pi_{2}^{L R} & =\frac{5 \sqrt{2}}{8} \frac{F_{1}^{L} F_{1}^{R *} m_{1}}{q^{2}+m_{1}^{2}}-\frac{\sqrt{5}}{2} \frac{F_{4}^{L} F_{4}^{R *} m_{4}}{q^{2}+m_{4}^{2}}
\end{array}\right\}
$$




\section{I-functions for next-to-minimal models}

The $\Pi$-functions for the next-to-minimal case are given for different representations in table 4. The $\mathrm{NMCHM}_{15_{\mathrm{L}}-1_{\mathrm{R}}}$ case is not included in the table because it cannot generate a Yukawa term in the Lagrangian.

\begin{tabular}{|c|c|c|c|}
\hline Models & $\Pi_{t_{L}}(h, \eta)$ & $\Pi_{t_{R}}(h, \eta)$ & $\Pi_{t_{L} t_{R}}(h, \eta)$ \\
\hline $\mathrm{NMCHM}_{6_{\mathrm{L}}-1_{\mathrm{R}}}$ & $\Pi_{0}^{L}+\Pi_{1}^{L} \frac{h^{2}}{f^{2}}$ & $\Pi_{0}^{R}$ & $\Pi_{1}^{L R} \frac{h}{f}$ \\
\hline $\mathrm{NMCHM}_{6_{\mathrm{L}}-6_{\mathrm{R}}}$ & $\Pi_{0}^{L}+\Pi_{1}^{L} \frac{h^{2}}{f^{2}}$ & $\Pi_{0}^{R}+\Pi_{1}^{R} \frac{h^{2}}{f^{2}}+\Pi_{\eta}^{R} \frac{\eta^{2}}{f^{2}}$ & $\Pi_{1}^{L R} \frac{h}{f} \sqrt{1-\frac{h^{2}}{f^{2}}-\frac{\eta^{2}}{f^{2}}}$ \\
\hline $\mathrm{NMCHM}_{6_{\mathrm{L}}-15_{\mathrm{R}}}$ & $\Pi_{0}^{L}+\Pi_{1}^{L} \frac{h^{2}}{f^{2}}$ & $\Pi_{0}^{R}+\Pi_{1}^{R} \frac{h^{2}}{f^{2}}$ & $\Pi_{1}^{L R} \frac{h}{f}$ \\
\hline $\mathrm{NMCHM}_{6_{\mathrm{L}}-20_{\mathrm{R}}}$ & $\Pi_{0}^{L}+\Pi_{1}^{L} \frac{h^{2}}{f^{2}}$ & $\Pi_{0}^{R}+\Pi_{1}^{R} \frac{h^{2}}{f^{2}}+\Pi_{2}^{R} \frac{h^{4}}{f^{4}}$ & $\frac{h}{f}\left(\Pi_{1}^{L R}+\Pi_{2}^{L R} \frac{h^{2}}{f^{2}}\right)$ \\
\hline $\mathrm{NMCHM}_{15_{\mathrm{L}}-6_{\mathrm{R}}}$ & $\Pi_{0}^{L}+\Pi_{1}^{L} \frac{h^{2}}{f^{2}}+\Pi_{\eta}^{L} \frac{\eta^{2}}{f^{2}}$ & $\Pi_{0}^{R}+\Pi_{1}^{R} \frac{h^{2}}{f^{2}}+\Pi_{\eta}^{R} \frac{\eta^{2}}{f^{2}}$ & $\Pi_{1}^{L R} \frac{h}{f}$ \\
\hline $\mathrm{NMCHM}_{15_{L}-15_{\mathrm{R}}}$ & $\Pi_{0}^{L}+\Pi_{1}^{L} \frac{h^{2}}{f^{2}}+\Pi_{\eta}^{L} \frac{\eta^{2}}{f^{2}}$ & $\Pi_{0}^{R}+\Pi_{1}^{R} \frac{h^{2}}{f^{2}}$ & $\Pi_{1}^{L R} \frac{h}{f} \sqrt{1-\frac{h^{2}}{f^{2}}-\frac{\eta^{2}}{f^{2}}}$ \\
\hline $\mathrm{NMCHM}_{15_{\mathrm{L}}-20_{\mathrm{R}}}$ & $\Pi_{0}^{L}+\Pi_{1}^{L} \frac{h^{2}}{f^{2}}+\Pi_{\eta}^{L} \frac{\eta^{2}}{f^{2}}$ & $\Pi_{0}^{R}+\Pi_{1}^{R} \frac{h^{2}}{f^{2}}+\Pi_{2}^{R} \frac{h^{4}}{f^{4}}$ & $\Pi_{1}^{L R} \frac{h}{f} \sqrt{1-\frac{h^{2}}{f^{2}}-\frac{\eta^{2}}{f^{2}}}$ \\
\hline $\mathrm{NMCHM}_{20_{L}-1_{R}}$ & $\begin{array}{l}\Pi_{0}^{L}+\Pi_{1}^{L} \frac{h^{2}}{f^{2}}+\Pi_{2}^{L} \frac{h^{4}}{f^{4}} \\
+\Pi_{\eta}^{L} \frac{\eta^{2}}{f^{2}}+\Pi_{h \eta}^{L} \frac{h^{2}}{f^{2}} \frac{\eta^{2}}{f^{2}}\end{array}$ & $\Pi_{0}^{R}$ & $\Pi_{1}^{L R} \frac{h}{f} \sqrt{1-\frac{h^{2}}{f^{2}}-\frac{\eta^{2}}{f^{2}}}$ \\
\hline $\mathrm{NMCHM}_{20_{\mathrm{L}}-6_{\mathrm{R}}}$ & $\begin{array}{l}\Pi_{0}^{L}+\Pi_{1}^{L} \frac{h^{2}}{f^{2}}+\Pi_{2}^{L} \frac{h^{4}}{f^{4}} \\
+\Pi_{\eta}^{L} \frac{\eta^{2}}{f^{2}}+\Pi_{h \eta}^{L} \frac{h^{2}}{f^{2}} \frac{\eta^{2}}{f^{2}}\end{array}$ & $\Pi_{0}^{R}+\Pi_{1}^{R} \frac{h^{2}}{f^{2}}+\Pi_{\eta}^{R} \frac{\eta^{2}}{f^{2}}$ & $\frac{h}{f}\left(\Pi_{1}^{L R}+\Pi_{2}^{L R} \frac{h^{2}}{f^{2}}+\Pi_{\eta}^{L R} \frac{\eta^{2}}{f^{2}}\right)$ \\
\hline $\mathrm{NMCHM}_{20_{\mathrm{L}}-15_{\mathrm{R}}}$ & $\begin{array}{l}\Pi_{0}^{L}+\Pi_{1}^{L} \frac{h^{2}}{f^{2}}+\Pi_{2}^{L} \frac{h^{4}}{f^{4}} \\
+\Pi_{\eta}^{L} \frac{\eta^{2}}{f^{2}}+\Pi_{h \eta}^{L} \frac{h^{2}}{f^{2}} \frac{\eta^{2}}{f^{2}}\end{array}$ & $\Pi_{0}^{R}+\Pi_{1}^{R} \frac{h^{2}}{f^{2}}$ & $\Pi_{1}^{L R} \frac{h}{f} \sqrt{1-\frac{h^{2}}{f^{2}}-\frac{\eta^{2}}{f^{2}}}$ \\
\hline $\mathrm{NMCHM}_{20_{L}-20_{R}}$ & $\begin{array}{l}\Pi_{0}^{L}+\Pi_{1}^{L} \frac{h^{2}}{f^{2}}+\Pi_{2}^{L} \frac{h^{4}}{f^{4}} \\
+\Pi_{\eta}^{L} \frac{\eta^{2}}{f^{2}}+\Pi_{h \eta}^{L} \frac{h^{2}}{f^{2}} \frac{\eta^{2}}{f^{2}}\end{array}$ & $\Pi_{0}^{R}+\Pi_{1}^{R} \frac{h^{2}}{f^{2}}+\Pi_{2}^{R} \frac{h^{4}}{f^{4}}$ & $\frac{h}{f} \sqrt{1-\frac{h^{2}}{f^{2}}-\frac{\eta^{2}}{f^{2}}}\left(\Pi_{1}^{L R}+\Pi_{2}^{L R} \frac{h^{2}}{f^{2}}\right)$ \\
\hline
\end{tabular}

Table 4. List of $\Pi$-functions for different representations of next-to-minimal model.

\section{Expressions for $\Delta_{t}, \delta_{t}$ and $\Delta_{t}^{\eta}$}

In table 5 we list the expressions for $\Delta_{t}$ and $\delta_{t}$ for the models $\mathrm{MCHM}_{5_{\mathrm{L}}-5_{\mathrm{R}}}, \mathrm{MCHM}_{14_{\mathrm{L}}-14_{\mathrm{R}}}$, $\mathrm{MCHM}_{14_{\mathrm{L}}-5_{\mathrm{R}}}$ and $\mathrm{MCHM}_{5_{\mathrm{L}}-14_{\mathrm{R}}}$, respectively.

\begin{tabular}{|c|c|c|}
\hline \multicolumn{1}{|c|}{ Models } & $\Delta_{t}$ & $\delta_{t}$ \\
\hline $\mathrm{MCHM}_{5_{\mathrm{L}}-5_{\mathrm{R}}}$ & $-\frac{3}{2}$ & $-\left(\frac{\Pi_{1}^{L}}{\Pi_{0}^{L}}+\frac{\Pi_{1}^{R}}{\Pi_{0}^{R}}\right)$ \\
\hline $\mathrm{MCHM}_{14_{\mathrm{L}}-14_{\mathrm{R}}}$ & $2 \frac{\Pi_{2}^{L R}}{\Pi_{1}^{L R}}-\frac{3}{2}$ & $-\left(\frac{\Pi_{1}^{L}}{\Pi_{0}^{L}}+\frac{\Pi_{1}^{R}}{\Pi_{0}^{R}}\right)$ \\
\hline $\mathrm{MCHM}_{14_{\mathrm{L}}-5_{\mathrm{R}}}$ & $2 \frac{\Pi_{2}^{L R}}{\Pi_{1}^{L R}}-\frac{1}{2}$ & $-\left(\frac{\Pi_{1}^{L}}{\Pi_{0}^{L}}+\frac{\Pi_{1}^{R}}{\Pi_{0}^{R}}\right)$ \\
\hline $\mathrm{MCHM}_{5_{\mathrm{L}}-14_{\mathrm{R}}}$ & $2 \frac{\Pi_{2}^{L R}}{\Pi_{1}^{L R}}-\frac{1}{2}$ & $-\left(\frac{\Pi_{1}^{L}}{\Pi_{0}^{L}}+\frac{\Pi_{1}^{R}}{\Pi_{0}^{R}}\right)$ \\
\hline
\end{tabular}

Table 5. Expressions for $\Delta_{t}$ and $\delta_{t}$ for different representations of $\mathrm{SO}(5)$ in which top quark is embedded. 
We present the expressions for $\left(\Delta_{t}+\delta_{t}\right)$ and $\Delta_{t}^{\eta}$, as defined in eq. (2.12), in terms of the form factors, for different $\mathrm{SO}(6)$ representations in table 6 .

\begin{tabular}{|c|c|c|}
\hline Models & & Coupling Modifiers \\
\hline \multirow{2}{*}{$\mathrm{NMCHM}_{6_{L}-1_{R}}$} & $\Delta_{t}+\delta_{t}$ & $-\left(\frac{\Pi_{1}^{L}}{\Pi_{0}^{L}}+\frac{1}{2}\right)$ \\
\hline & $\Delta_{t}^{\eta}$ & 0 \\
\hline \multirow[t]{2}{*}{$\mathrm{NMCHM}_{6_{\mathrm{L}}-6_{\mathrm{R}}}$} & $\Delta_{t}+\delta_{t}$ & $-\left(1+\frac{\Pi_{n}^{R}}{\Pi_{0}^{R}} \chi\right)^{-1}\left[\frac{\Pi_{1}^{L}}{\Pi_{0}^{L}}+\frac{\Pi_{1}^{R}}{\Pi_{0}^{R}}+\frac{3}{2}+\left(\frac{\Pi_{1}^{L}}{\Pi_{0}^{L}} \frac{\Pi_{n}^{R}}{\Pi_{0}^{R}}+\frac{1}{2} \frac{\Pi_{n}^{R}}{\Pi_{0}^{R}}\right) \chi\right]$ \\
\hline & $\Delta_{t}^{\eta}$ & $\left(1+\frac{\Pi_{\eta}^{R}}{\Pi_{0}^{R}}\right)\left(1+\frac{\Pi_{\eta}^{R}}{\Pi_{0}^{R}} \chi\right)^{-1} \sqrt{\frac{\chi}{1-\chi}}$ \\
\hline \multirow[t]{2}{*}{$\mathrm{NMCHM}_{6_{\mathrm{L}}-15_{\mathrm{R}}}$} & $\Delta_{t}+\delta_{t}$ & $-\left[\frac{\Pi_{1}^{L}}{\Pi_{0}^{L}}+\frac{\Pi_{1}^{R}}{\Pi_{0}^{R}}+\frac{1}{2}\right]$ \\
\hline & $\Delta_{t}^{\eta}$ & 0 \\
\hline \multirow[t]{2}{*}{$\mathrm{NMCHM}_{6_{\mathrm{L}}-20_{\mathrm{R}}}$} & $\Delta_{t}+\delta_{t}$ & {$\left[2 \frac{\Pi_{2}^{L R}}{\Pi_{1}^{L R}}-\frac{\Pi_{1}^{L}}{\Pi_{0}^{L}}-\frac{\Pi_{1}^{R}}{\Pi_{0}^{R}}-\frac{1}{2}\right]$} \\
\hline & $\Delta_{t}^{\eta}$ & 0 \\
\hline \multirow[t]{2}{*}{$\mathrm{NMCHM}_{15_{\mathrm{L}}-6_{\mathrm{R}}}$} & $\Delta_{t}+\delta_{t}$ & $\begin{array}{l}-\left(1+\frac{\Pi_{\eta}^{L}}{\Pi_{0}^{L}} \chi\right)^{-1}\left(1+\frac{\Pi_{\eta}^{R}}{\Pi_{0}^{R}} \chi\right)^{-1}\left[\frac{\Pi_{1}^{L}}{\Pi_{0}^{L}}+\frac{\Pi_{1}^{R}}{\Pi_{0}^{R}}+\frac{1}{2}\right. \\
\left.-\left(\frac{1}{2} \frac{\Pi_{n}^{L}}{\Pi_{0}^{L}}+\frac{1}{2} \frac{\Pi_{n}^{R}}{\Pi_{0}^{R}}-\frac{\Pi_{1}^{L}}{\Pi_{0}^{L}} \frac{\Pi_{\eta}^{R}}{\Pi_{0}^{R}}-\frac{\Pi_{1}^{R}}{\Pi_{0}^{R}} \frac{\Pi_{\eta}^{L}}{\Pi_{0}^{L}}\right) \chi-\frac{3}{2} \frac{\Pi_{n}^{L}}{\Pi_{0}^{L}} \frac{\Pi_{n}^{R}}{\Pi_{0}^{R}} \chi^{2}\right]\end{array}$ \\
\hline & $\Delta_{t}^{\eta}$ & $(1-\chi)\left(1+\frac{\Pi_{n}^{L}}{\Pi_{0}^{L}} \chi\right)^{-1}\left(1+\frac{\Pi_{n}^{R}}{\Pi_{0}^{R}} \chi\right)^{-1}\left[\frac{\Pi_{n}^{L}}{\Pi_{0}^{L}}+\frac{\Pi_{n}^{R}}{\Pi_{0}^{R}}+2 \frac{\Pi_{\eta}^{L}}{\Pi_{0}^{L}} \frac{\Pi_{n}^{R}}{\Pi_{0}^{R}} \chi\right] \sqrt{\frac{\chi}{1-\chi}}$ \\
\hline \multirow[t]{2}{*}{$\mathrm{NMCHM}_{15_{L}-15_{R}}$} & $\Delta_{t}+\delta_{t}$ & $-\left(1+\frac{\Pi_{\eta}^{L}}{\Pi_{0}^{L}} \chi\right)^{-1}\left[\frac{\Pi_{1}^{L}}{\Pi_{0}^{L}}+\frac{\Pi_{1}^{R}}{\Pi_{0}^{R}}+\frac{3}{2}+\left(\frac{1}{2} \frac{\Pi_{\eta}^{L}}{\Pi_{0}^{L}}+\frac{\Pi_{n}^{L}}{\Pi_{0}^{L}} \frac{\Pi_{1}^{R}}{\Pi_{0}^{R}}\right) \chi\right]$ \\
\hline & $\Delta_{t}^{\eta}$ & $\left(1+\frac{\Pi_{\eta}^{L}}{\Pi_{0}^{L}}\right)\left(1+\frac{\Pi_{\eta}^{L}}{\Pi_{0}^{L}} \chi\right)^{-1} \sqrt{\frac{\chi}{1-\chi}}$ \\
\hline \multirow[t]{2}{*}{$\mathrm{NMCHM}_{15_{\mathrm{L}}-20_{\mathrm{R}}}$} & $\Delta_{t}+\delta_{t}$ & $-\left(1+\frac{\Pi_{\eta}^{L}}{\Pi_{0}^{L}} \chi\right)^{-1}\left[\frac{\Pi_{1}^{L}}{\Pi_{0}^{L}}+\frac{\Pi_{1}^{R}}{\Pi_{0}^{R}}+\frac{3}{2}+\left(\frac{1}{2} \frac{\Pi_{\eta}^{L}}{\Pi_{0}^{L}}+\frac{\Pi_{\eta}^{L}}{\Pi_{0}^{L}} \frac{\Pi_{1}^{R}}{\Pi_{0}^{R}}\right) \chi\right]$ \\
\hline & $\Delta_{t}^{\eta}$ & $\left(1+\frac{\Pi_{\eta}^{L}}{\Pi_{0}^{L}}\right)\left(1+\frac{\Pi_{n}^{L}}{\Pi_{0}^{L}} \chi\right)^{-1} \sqrt{\frac{\chi}{1-\chi}}$ \\
\hline \multirow[t]{2}{*}{$\mathrm{NMCHM}_{20_{\mathrm{L}}-1_{\mathrm{R}}}$} & $\Delta_{t}+\delta_{t}$ & $-\left(1+\frac{\Pi_{\eta}^{L}}{\Pi_{0}^{L}} \chi\right)^{-1}\left[\frac{\Pi_{1}^{L}}{\Pi_{0}^{L}}+\frac{3}{2}+\left(\frac{1}{2} \frac{\Pi_{\eta}^{L}}{\Pi_{0}^{L}}+\frac{\Pi_{h \eta}^{L}}{\Pi_{0}^{L}}\right) \chi\right]$ \\
\hline & $\Delta_{t}^{\eta}$ & $\left(1+\frac{\Pi_{n}^{L}}{\Pi_{0}^{L}}\right)\left(1+\frac{\Pi_{n}^{L}}{\Pi_{0}^{L}} \chi\right)^{-1} \sqrt{\frac{\chi}{1-\chi}}$ \\
\hline \multirow[t]{2}{*}{$\mathrm{NMCHM}_{20_{\mathrm{L}}-6_{\mathrm{R}}}$} & $\Delta_{t}+\delta_{t}$ & 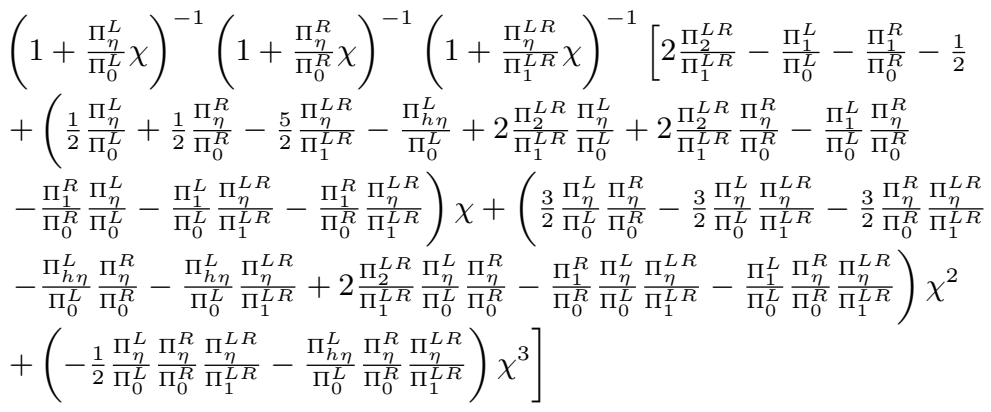 \\
\hline & $\Delta_{t}^{\eta}$ & $\begin{array}{l}-(1-\chi)\left(1+\frac{\Pi_{n}^{L}}{\Pi_{0}^{L}} \chi\right)^{-1}\left(1+\frac{\Pi_{n}^{R}}{\Pi_{0}^{R}} \chi\right)^{-1}\left(1+\frac{\Pi_{n}^{L R}}{\Pi_{1}^{L R}} \chi\right)^{-1} \\
{\left[2 \frac{\Pi_{n}^{L R}}{\Pi_{1}^{L R}}-\frac{\Pi_{n}^{L}}{\Pi_{0}^{L}}-\frac{\Pi_{n}^{R}}{\Pi_{0}^{R}}+\left(\frac{\Pi_{n}^{L}}{\Pi_{0}^{L}} \frac{\Pi_{n}^{L R}}{\Pi_{1}^{L R}}+\frac{\Pi_{n}^{R}}{\Pi_{0}^{R}} \frac{\Pi_{n}^{L R}}{\Pi_{1}^{L R}}-2 \frac{\Pi_{n}^{L}}{\Pi_{0}^{L}} \frac{\Pi_{n}^{R}}{\Pi_{0}^{R}}\right) \chi\right] \sqrt{\frac{\chi}{1-\chi}}}\end{array}$ \\
\hline
\end{tabular}




\begin{tabular}{|c|c|c|}
\hline \multirow[t]{2}{*}{$\mathrm{NMCHM}_{20_{\mathrm{L}}-15_{\mathrm{R}}}$} & $\Delta_{t}+\delta_{t}$ & $-\left(1+\frac{\Pi_{n}^{L}}{\Pi_{0}^{L}} \chi\right)^{-1}\left[\frac{\Pi_{1}^{L}}{\Pi_{0}^{L}}+\frac{\Pi_{1}^{R}}{\Pi_{0}^{R}}+\frac{3}{2}+\left(\frac{1}{2} \frac{\Pi_{n}^{L}}{\Pi_{0}^{L}}+\frac{\Pi_{h}^{L}}{\Pi_{0}^{L}}+\frac{\Pi_{n}^{L}}{\Pi_{0}^{L}} \frac{\Pi_{1}^{R}}{\Pi_{0}^{R}}\right) \chi\right]$ \\
\hline & $\Delta_{t}^{\eta}$ & $\left(1+\frac{\Pi_{\eta}^{L}}{\Pi_{0}^{L}}\right)\left(1+\frac{\Pi_{\eta}^{L}}{\Pi_{0}^{L}} \chi\right)^{-1} \sqrt{\frac{\chi}{1-\chi}}$ \\
\hline \multirow[t]{2}{*}{$\mathrm{NMCHM}_{20_{\mathrm{L}}-20_{\mathrm{R}}}$} & $\Delta_{t}+\delta_{t}$ & $\begin{array}{l}\left(1+\frac{\Pi_{\eta}^{L}}{\Pi_{0}^{L}} \chi\right)^{-1}\left[2 \frac{\Pi_{2}^{L} R}{\Pi_{1}^{L R}}-\frac{\Pi_{1}^{L}}{\Pi_{0}^{L}}-\frac{\Pi_{1}^{R}}{\Pi_{0}^{R}}-\frac{3}{2}\right. \\
\left.+\left(2 \frac{\Pi_{2}^{L} R}{\Pi_{1}^{L R}} \frac{\Pi_{\eta}^{L}}{\Pi_{0}^{L}}-\frac{\Pi_{1}^{R}}{\Pi_{0}^{R}} \frac{\Pi_{\eta}^{L}}{\Pi_{0}^{L}}-\frac{1}{2} \frac{\Pi_{\eta}^{L}}{\Pi_{0}^{L}}-\frac{\Pi_{h \eta}^{L}}{\Pi_{0}^{L}}\right) \chi\right]\end{array}$ \\
\hline & $\Delta_{t}^{\eta}$ & $\left(1+\frac{\Pi_{\eta}^{L}}{\Pi_{0}^{L}}\right)\left(1+\frac{\Pi_{\eta}^{L}}{\Pi_{0}^{L}} \chi\right)^{-1} \sqrt{\frac{\chi}{1-\chi}}$ \\
\hline
\end{tabular}

Table 6. Expressions for $\left(\Delta_{t}+\delta_{t}\right)$ and $\Delta_{t}^{\eta}$ for different representations of $\mathrm{SO}(6)$ in which the top quark is embedded.

Open Access. This article is distributed under the terms of the Creative Commons Attribution License (CC-BY 4.0), which permits any use, distribution and reproduction in any medium, provided the original author(s) and source are credited.

\section{References}

[1] D.B. Kaplan and H. Georgi, SU(2) $\times$ U(1) Breaking by Vacuum Misalignment, Phys. Lett. B 136 (1984) 183 [INSPIRE].

[2] M.J. Dugan, H. Georgi and D.B. Kaplan, Anatomy of a Composite Higgs Model, Nucl. Phys. B 254 (1985) 299 [INSPIRE].

[3] R. Contino, The Higgs as a Composite Nambu-Goldstone Boson, in Physics of the large and the small, TASI 09, proceedings of the Theoretical Advanced Study Institute in Elementary Particle Physics, Boulder, Colorado, U.S.A., 1-26 June 2009, pp. 235-306 (2011) DOI:10.1142/9789814327183_0005 [arXiv: 1005.4269] [INSPIRE].

[4] G. Panico and A. Wulzer, The Composite Nambu-Goldstone Higgs, Lect. Notes Phys. 913 (2016) pp.1 [arXiv: 1506. 01961] [INSPIRE].

[5] C. Csáki and P. Tanedo, Beyond the Standard Model, in Proceedings, 2013 European School of High-Energy Physics (ESHEP 2013), Paradfurdo, Hungary, June 5-18, 2013, pp. 169-268 (2015) DOI:10.5170/CERN-2015-004.169 [arXiv: 1602.04228] [INSPIRE].

[6] R. Contino, Y. Nomura and A. Pomarol, Higgs as a holographic pseudoGoldstone boson, Nucl. Phys. B 671 (2003) 148 [hep-ph/0306259] [InSPIRE].

[7] R. Contino, T. Kramer, M. Son and R. Sundrum, Warped/composite phenomenology simplified, JHEP 05 (2007) 074 [hep-ph/0612180] [INSPIRE].

[8] C. Csáki, M. Geller and O. Telem, Tree-level Quartic for a Holographic Composite Higgs, arXiv: 1710.08921 [INSPIRE].

[9] D. Espriu and A. Katanaeva, Holographic description of $\mathrm{SO}(5) \rightarrow \mathrm{SO}(4)$ composite Higgs model, arXiv:1706.02651 [INSPIRE].

[10] A. De Simone, O. Matsedonskyi, R. Rattazzi and A. Wulzer, A First Top Partner Hunter's Guide, JHEP 04 (2013) 004 [arXiv:1211.5663] [INSPIRE]. 
[11] A. Thamm, R. Torre and A. Wulzer, Future tests of Higgs compositeness: direct vs indirect, JHEP 07 (2015) 100 [arXiv: 1502.01701] [INSPIRE].

[12] A. Azatov, D. Chowdhury, D. Ghosh and T.S. Ray, Same sign di-lepton candles of the composite gluons, JHEP 08 (2015) 140 [arXiv:1505.01506] [INSPIRE].

[13] J.P. Araque, N.F. Castro and J. Santiago, Interpretation of Vector-like Quark Searches: Heavy Gluons in Composite Higgs Models, JHEP 11 (2015) 120 [arXiv:1507.05628] [INSPIRE].

[14] ATLAS collaboration, Search for pair production of vector-like top quarks in events with one lepton, jets and missing transverse momentum in $\sqrt{s}=13 \mathrm{TeV}$ pp collisions with the ATLAS detector, JHEP 08 (2017) 052 [arXiv:1705.10751] [INSPIRE].

[15] CMS collaboration, Search for top quark partners with charge 5/3 in proton-proton collisions at $\sqrt{s}=13 \mathrm{TeV}$, JHEP 08 (2017) 073 [arXiv: 1705.10967] [INSPIRE].

[16] CMS collaboration, Search for pair production of vector-like $T$ and B quarks in single-lepton final states using boosted jet substructure in proton-proton collisions at $\sqrt{s}=13 \mathrm{TeV}$, JHEP 11 (2017) 085 [arXiv:1706.03408] [INSPIRE].

[17] M. Chala, Direct bounds on heavy toplike quarks with standard and exotic decays, Phys. Rev. D 96 (2017) 015028 [arXiv: 1705.03013] [INSPIRE].

[18] J. Yepes and A. Zerwekh, Top partner-resonance interplay in a composite Higgs framework, arXiv:1711.10523 [INSPIRE].

[19] D. Pappadopulo, A. Thamm and R. Torre, A minimally tuned composite Higgs model from an extra dimension, JHEP 07 (2013) 058 [arXiv: 1303.3062] [INSPIRE].

[20] D. Croon, B.M. Dillon, S.J. Huber and V. Sanz, Exploring holographic Composite Higgs models, JHEP 07 (2016) 072 [arXiv:1510.08482] [INSPIRE].

[21] A. Banerjee, G. Bhattacharyya and T.S. Ray, Improving Fine-tuning in Composite Higgs Models, Phys. Rev. D 96 (2017) 035040 [arXiv:1703.08011] [InSPIRE].

[22] C. Csáki, T. Ma and J. Shu, Trigonometric Parity for the Composite Higgs, arXiv: 1709.08636 [INSPIRE].

[23] Z. Chacko, H.-S. Goh and R. Harnik, The Twin Higgs: Natural electroweak breaking from mirror symmetry, Phys. Rev. Lett. 96 (2006) 231802 [hep-ph/0506256] [INSPIRE].

[24] K. Agashe, R. Contino and A. Pomarol, The Minimal composite Higgs model, Nucl. Phys. B 719 (2005) 165 [hep-ph/0412089] [INSPIRE].

[25] G. Panico and A. Wulzer, The Discrete Composite Higgs Model, JHEP 09 (2011) 135 [arXiv:1106.2719] [INSPIRE].

[26] O. Matsedonskyi, G. Panico and A. Wulzer, Light Top Partners for a Light Composite Higgs, JHEP 01 (2013) 164 [arXiv: 1204.6333] [INSPIRE].

[27] D. Marzocca, M. Serone and J. Shu, General Composite Higgs Models, JHEP 08 (2012) 013 [arXiv: 1205.0770] [INSPIRE].

[28] A. Pomarol and F. Riva, The Composite Higgs and Light Resonance Connection, JHEP 08 (2012) 135 [arXiv: 1205.6434] [INSPIRE].

[29] M. Carena, L. Da Rold and E. Pontón, Minimal Composite Higgs Models at the LHC, JHEP 06 (2014) 159 [arXiv: 1402.2987] [INSPIRE]. 
[30] G. Panico, M. Redi, A. Tesi and A. Wulzer, On the Tuning and the Mass of the Composite Higgs, JHEP 03 (2013) 051 [arXiv:1210.7114] [InSPIRE].

[31] M. Montull, F. Riva, E. Salvioni and R. Torre, Higgs Couplings in Composite Models, Phys. Rev. D 88 (2013) 095006 [arXiv: 1308.0559] [INSPIRE].

[32] A. Carmona and F. Goertz, A naturally light Higgs without light Top Partners, JHEP 05 (2015) 002 [arXiv:1410.8555] [INSPIRE].

[33] S. Kanemura, K. Kaneta, N. Machida, S. Odori and T. Shindou, Single and double production of the Higgs boson at hadron and lepton colliders in minimal composite Higgs models, Phys. Rev. D 94 (2016) 015028 [arXiv: 1603.05588] [inSPIRE].

[34] M.B. Gavela, K. Kanshin, P.A.N. Machado and S. Saa, The linear-non-linear frontier for the Goldstone Higgs, Eur. Phys. J. C 76 (2016) 690 [arXiv:1610.08083] [INSPIRE].

[35] D. Liu, I. Low and C.E.M. Wagner, Modification of Higgs Couplings in Minimal Composite Models, Phys. Rev. D 96 (2017) 035013 [arXiv:1703.07791] [InSPIRE].

[36] B. Gripaios, A. Pomarol, F. Riva and J. Serra, Beyond the Minimal Composite Higgs Model, JHEP 04 (2009) 070 [arXiv: 0902.1483] [INSPIRE].

[37] M. Redi and A. Tesi, Implications of a Light Higgs in Composite Models, JHEP 10 (2012) 166 [arXiv: 1205.0232] [INSPIRE].

[38] J. Barnard, T. Gherghetta and T.S. Ray, UV descriptions of composite Higgs models without elementary scalars, JHEP 02 (2014) 002 [arXiv: 1311.6562] [INSPIRE].

[39] J. Serra, Beyond the Minimal Top Partner Decay, JHEP 09 (2015) 176 [arXiv:1506.05110] [INSPIRE].

[40] M. Low, A. Tesi and L.-T. Wang, A pseudoscalar decaying to photon pairs in the early LHC Run 2 data, JHEP 03 (2016) 108 [arXiv: 1512.05328] [INSPIRE].

[41] H. Cai, T. Flacke and M. Lespinasse, A composite scalar hint from di-boson resonances?, arXiv: 1512.04508 [INSPIRE].

[42] A. Arbey, G. Cacciapaglia, H. Cai, A. Deandrea, S. Le Corre and F. Sannino, Fundamental Composite Electroweak Dynamics: Status at the LHC, Phys. Rev. D 95 (2017) 015028 [arXiv: 1502.04718] [INSPIRE].

[43] C. Niehoff, P. Stangl and D.M. Straub, Electroweak symmetry breaking and collider signatures in the next-to-minimal composite Higgs model, JHEP 04 (2017) 117 [arXiv: 1611.09356] [INSPIRE].

[44] V. Sanz and J. Setford, Composite Higgs models after Run2, arXiv:1703.10190 [INSPIRE].

[45] G.F. Giudice, C. Grojean, A. Pomarol and R. Rattazzi, The Strongly-Interacting Light Higgs, JHEP 06 (2007) 045 [hep-ph/0703164] [INSPIRE].

[46] R. Contino, M. Ghezzi, C. Grojean, M. Muhlleitner and M. Spira, Effective Lagrangian for a light Higgs-like scalar, JHEP 07 (2013) 035 [arXiv: 1303.3876] [INSPIRE].

[47] M. Hashimoto, Revisiting vectorlike quark models with enhanced top Yukawa coupling, Phys. Rev. D 96 (2017) 035020 [arXiv:1704.02615] [INSPIRE].

[48] S. Jana and S. Nandi, New Physics Scale from Higgs Observables with Effective Dimension-6 Operators, arXiv: 1710.00619 [INSPIRE]. 
[49] G. Cacciapaglia, A. Deandrea, G. Drieu La Rochelle and J.-B. Flament, Higgs couplings beyond the Standard Model, JHEP 03 (2013) 029 [arXiv: 1210.8120] [INSPIRE].

[50] A. Falkowski, F. Riva and A. Urbano, Higgs at last, JHEP 11 (2013) 111 [arXiv:1303.1812] [INSPIRE].

[51] A. Azatov, R. Contino, A. Di Iura and J. Galloway, New Prospects for Higgs Compositeness in $h \rightarrow Z \gamma$, Phys. Rev. D 88 (2013) 075019 [arXiv: 1308.2676] [INSPIRE].

[52] S.R. Coleman and E.J. Weinberg, Radiative Corrections as the Origin of Spontaneous Symmetry Breaking, Phys. Rev. D 7 (1973) 1888 [InSPIRE].

[53] ATLAS, CMS collaborations, Measurements of the Higgs boson production and decay rates and constraints on its couplings from a combined ATLAS and CMS analysis of the LHC pp collision data at $\sqrt{s}=7$ and 8 TeV, JHEP 08 (2016) 045 [arXiv:1606.02266] [INSPIRE].

[54] ATLAS collaboration, Measurements of the Higgs boson production cross section via Vector Boson Fusion and associated $W H$ production in the $W W^{*} \rightarrow \ell \nu \ell \nu$ decay mode with the ATLAS detector at $\sqrt{s}=13 \mathrm{TeV}$, ATLAS-CONF-2016-112 (2016).

[55] ATLAS collaboration, Measurements of Higgs boson properties in the diphoton decay channel with $36.1 \mathrm{fb}^{-1} \mathrm{pp}$ collision data at the center-of-mass energy of $13 \mathrm{TeV}$ with the ATLAS detector, ATLAS-CONF-2017-045 (2017).

[56] ATLAS collaboration, Evidence for the associated production of the Higgs boson and a top quark pair with the ATLAS detector, ATLAS-CONF-2017-077 (2017).

[57] ATLAS collaboration, Measurement of the Higgs boson coupling properties in the $H \rightarrow Z Z^{*} \rightarrow 4 \ell$ decay channel at $\sqrt{s}=13 \mathrm{TeV}$ with the ATLAS detector, ATLAS-CONF-2017-043 (2017).

[58] ATLAS collaboration, Evidence for the $H \rightarrow b \bar{b}$ decay with the ATLAS detector, JHEP 12 (2017) 024 [arXiv: 1708.03299] [INSPIRE].

[59] ATLAS collaboration, Search for the Standard Model Higgs boson produced in association

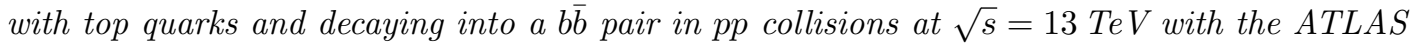
detector, ATLAS-CONF-2017-076 (2017).

[60] CMS collaboration, Higgs to WW measurements with $15.2 \mathrm{fb}^{-1}$ of $13 \mathrm{TeV}$ proton-proton collisions, CMS-PAS-HIG-16-021.

[61] CMS collaboration, Measurements of properties of the Higgs boson in the diphoton decay channel with the full 2016 data set, CMS-PAS-HIG-16-040.

[62] CMS collaboration, Measurements of properties of the Higgs boson decaying into the four-lepton final state in pp collisions at $\sqrt{s}=13 \mathrm{TeV}$, JHEP 11 (2017) 047 [arXiv: 1706.09936] [INSPIRE].

[63] CMS collaboration, Observation of the Higgs boson decay to a pair of $\tau$ leptons with the CMS detector, Phys. Lett. B 779 (2018) 283 [arXiv:1708.00373] [INSPIRE].

[64] CMS collaboration, Evidence for the Higgs boson decay to a bottom quark-antiquark pair, arXiv: 1709.07497 [INSPIRE].

[65] CMS collaboration, Search for the associated production of a Higgs boson with a top quark pair in final states with a $\tau$ lepton at $\sqrt{s}=13 \mathrm{TeV}$, CMS-PAS-HIG-17-003.

[66] CMS collaboration, Search for Higgs boson production in association with top quarks in multilepton final states at $\sqrt{s}=13 \mathrm{TeV}$, CMS-PAS-HIG-17-004. 
[67] A. Azatov and J. Galloway, Light Custodians and Higgs Physics in Composite Models, Phys. Rev. D 85 (2012) 055013 [arXiv:1110.5646] [INSPIRE].

[68] M. Frigerio, J. Serra and A. Varagnolo, Composite GUTs: models and expectations at the LHC, JHEP 06 (2011) 029 [arXiv:1103.2997] [INSPIRE].

[69] J. Barnard, T. Gherghetta, T.S. Ray and A. Spray, The Unnatural Composite Higgs, JHEP 01 (2015) 067 [arXiv: 1409.7391] [InSPIRE].

[70] M. Frigerio, A. Pomarol, F. Riva and A. Urbano, Composite Scalar Dark Matter, JHEP 07 (2012) 015 [arXiv:1204.2808] [INSPIRE].

[71] D. Marzocca and A. Urbano, Composite Dark Matter and LHC Interplay, JHEP 07 (2014) 107 [arXiv: 1404.7419] [INSPIRE].

[72] N. Fonseca, R. Zukanovich Funchal, A. Lessa and L. Lopez-Honorez, Dark Matter Constraints on Composite Higgs Models, JHEP 06 (2015) 154 [arXiv:1501.05957] [INSPIRE].

[73] M. Kim, S.J. Lee and A. Parolini, WIMP Dark Matter in Composite Higgs Models and the Dilaton Portal, arXiv: 1602.05590 [INSPIRE].

[74] J. Ellis and T. You, Global Analysis of Experimental Constraints on a Possible Higgs-Like Particle with Mass $125 \mathrm{GeV}$, JHEP 06 (2012) 140 [arXiv:1204.0464] [INSPIRE].

[75] M. Chala, G. Durieux, C. Grojean, L. de Lima and O. Matsedonskyi, Minimally extended SILH, JHEP 06 (2017) 088 [arXiv:1703.10624] [INSPIRE]. 